\title{
LES BRYOZOAIRES CHEILOSTOMES DU NÉOGÈNE BÉTIQUE (SE ESPAGNE)
}

\author{
Simone POUYET \\ Centre des Sciences de la Terre et ERS 2042, Université Claude-Bernard Lyon \\ 1, F-69622 Villeurbanne Cedex.
}

Pouyet, S. 2000. Les bryozoaires cheilostomes du Néogène bétique (SE Espagne). [Cheilostome Bryozoa from the Betic Neogene (SE Spain).] Revista Española de Paleontología, 15(2), 181-202. ISSN 0213-6937.

\begin{abstract}
Bryozoa have been collected since 1960 from thirteen Neogene outcrops in South-East Spain. A hundred and four species of Cheilostomata are recognized, described and some of them figured, including one new species, Gephyrotes fortunensis. This is essentially a palaeontological update. Nevertheless some data are given about some of the outcrops. It is a first step towards a more complete palaeogeographical and palaeoecological study of the Betic Neogene.
\end{abstract}

Keywords: Bryozoa, Cheilostomata, Neogene, Systematics, Spain.

RÉSUMÉ

Depuis 1960, nombre de Bryozoaires ont été récoltés dans 13 gisements néogènes du Sud-Est de l'Espagne. Cent quatre espèces de Cheilostomes ont été reconnues, elles sont décrites et quelques-unes sont figurées. Une espèce nouvelle, Gephyrotes fortunensis, est définie. Il s'agit essentiellement d'un travail paléontologique, avec quelques données sur certains gisements. C'est une première étape vers une étude paléoécologique et paléogéographique des faunes du Néogène bétique.

Mots-clés: Bryozoa, Cheilostomata, Néogène, Systématique, Espagne.

\section{RESUMEN}

Desde 1960 se ha llevado a cabo la recogida de numerosos briozoos en 13 yacimientos neógenos del sureste de España. Se han reconocido ciento cuatro especies de Cheilostomata, que son referidas en este trabajo, en el que también se figuran algunas de ellas. Se define la nueva especie Gephyrotes fortunensis. El trabajo es fundamentalmente paleontológico, con algunos datos sobre ciertos yacimientos. Se trata de una primera etapa de un estudio paleoecológico y paleobiogeográfico más completo de las faunas del Neógeno bético.

\section{Palabras clave: Bryozoa, Cheilostomata, Neógeno, Sistemática, España.}

\section{INTRODUCTION}

Les gisements étudiés se situent dans la partie sud-est de la péninsule ibérique, zone bétique s'étendant d'Alicante à Alméria. Ils sont situés successivement du Nord au Sud dans les bassins d'Alicante, Fortuna, Murcie, Aguilas, Nijar et Carboneras (Fig. 1). Les récoltes ont été faites soit par Montenat (1960-1970), soit par David et Pouyet (1973), soit par Pouyet (1989).

La région étudiée forme un corridor d'environ $250 \mathrm{~km}$ de long et $70 \mathrm{~km}$ de large, très complexe du point de vue structural (Fig. 1). De nombreuses failles l'ont affecté lors de la collision Europe-Afrique d'où des phénomènes de déformation, dépôts de sédiments profonds, forte activité magmatique. Parmi les travaux les plus marquants des dernières années sur le Néogène terminal (du Tortonien au Pliocène), nous citerons ceux de Montenat (1977, 1990), Sanz de Galdeano (1990), Santisteban et Taberner (1983, bassin de Fortuna), de Michalzik (1997, Messinien), et pour l'aspect plus sédimentologique Brachert et al. (1996). Cette zone comprend de nombreux édifices récifaux (Fortuna, Santa Pola, etc).

L'âge des gisements miocènes est donné sous réserve, certains gisements étant considérés d'âge tantôt tortonien supérieur, tantôt messinien. Ainsi Montenat en 1977 utilisait les termes Tortonien II et Miocène terminal; en 


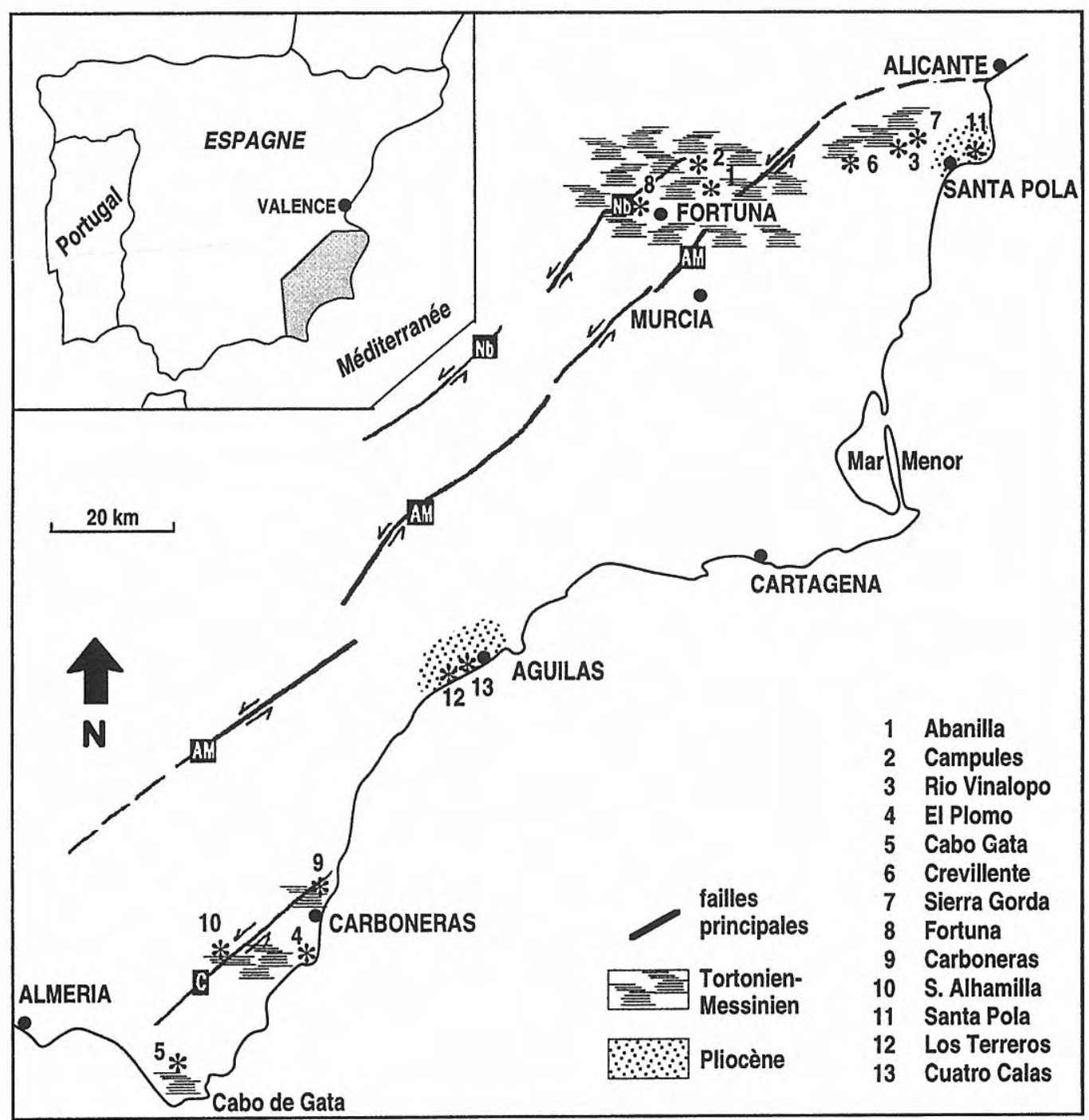

Figure 1. Localisation des gisements, d'après Michalzik (1997) simplifié. Failles principales: $C=$ Carboneras, $A M=$ Alhamilla de Murcia, $\mathrm{Nb}=$ Abanilla (faille nord bétique).

Map showing the distribution of the outcrops (simplified after Michalzik, 1997). Principal faults: C=Carboneras, $A M=$ Alhamilla de Murcia, $\mathrm{Nb}=$ Abanilla (North Betic Fault).

1990 le terme Miocène terminal est remplacé par Messinien sans que la superposition des termes soit absolue.

\section{ETUDE SYSTEMATIQUE}

Les travaux sur les faunes de bryozoaires du Néogène supérieur d'Espagne sont rares. Une liste avait été donnée en 1990 (Pouyet in Montenat) sans figurations, ni données sur les gisements. En 1992 dans le travail de Barrier et al. sur la faune de Carboneras une liste préliminaire est donnée généralement limitée aux genres. Un travail ancien (Reguant, 1961) donnait un liste très succinte. Le même auteur dans un travail plus récent (1993) sur le Pliocène de Huelva (SW Espagne) décrit de nombreuses espèces.

Le matériel étudié est déposé dans les collections de Géologie de 1'Université Claude-Bernard-Lyon1, il est inventorié sous le sigle FSL suivi d'un numéro d'inventaire; seuls les numéros des spécimens figurés sont indiqués; la liste complète peut être demandée au conservateur des Collections.

La deuxième édition du Traité de Moore sur les Cheilostomes n'étant pas encore éditée, nous avons tenu compte de travaux récents sur la systématique, notamment la liste préliminaire des genres et sous-genres (1995) distribuée au Congrès de l'International Bryozoology Association en Nouvelle -Zélande.

Pour chaque espèce, nous donnons la référence originale et une ou plusieurs références assez récentes incluant des descriptions et des illustrations permettant une meilleure appréhension de l'espèce. Nous avons favorisé les références concernant les domaines méditerranéen et atlantique nord-est. Une description n'est donnée que si l'espèce n'a pas été décrite dans les travaux de Moissette (1988), El Hajjaji (1992), HaddadiHamdane (1996) sur les faunes du Néogène récent 
d'Algérie et du Maroc: on y trouvera les descriptions et figurations détaillées de la plupart des formes trouvées en Espagne. Dans quelques cas, nous avons jugé bon de compléter ou préciser certains points.

\section{CLASSE GYMNOLAEMATA Allman, 1856 ORDRE CHEILOSTOMATIDA Busk, 1852 SOUS-ORDRE ANASCA Levinsen, 1909 Famille Membraniporidae Busk, 1854 Genre Biflustra d'Orbigny, 1852 Biflustra savarti (Savigny-Audouin, 1826)}

1826 Flustra Savartii Savigny-Audouin, 69, pl. 10, fig. 10. 1988 Biflustra savarti (Sav.-Aud.); Moissette, 73, pl. 11, fig. $6,9$.

Tortonien supérieur: El Plomo. Messinien: Sierra Alhamilla (basssin de Nijar). Pliocène: Cuatro Calas, Los Terreros, Santa Pola.

\section{Genre Conopeum Gray, 1848}

Conopeum reticulum (Linné, 1767)

1767 Millepora reticulum Linné, 1284.

1988 Conopeum reticulum (L.); Zabala et Maluquer, 76, fig. 65 ; pl. 1, fig. E.

1992 Conopeum reticulum (L.); El Hajjaji, 87, pl. 4, fig. 3.

Tortonien supérieur: Fortuna, Sierra Gorda.

Famille Electridae Lagaaij, 1952

Genre Electra Lamouroux, 1816

Electra monostachys (Busk, 1854)

1854 Membranipora monostachys Busk, 61, pl. 70, figs. 1-2. 1992 Electra monostachys (Busk); El Hajjaji, 88, pl. 4, fig. 1.

1997a Electra monostachys (Busk); Pouyet, 14, text-fig. 4.

Tortonien supérieur: Fortuna. Pliocène: Cuatro Calas.

Famille Hincksinidae Canu et Bassler, 1927

Genre Hincksina Norman, 1903

Hincksina flustroides (Hincks, 1877)

Fig. $2 \mathrm{~g}$

1877 Membranipora flustroides Hincks, 213.

1988 Hincksina flustroides (Hincks); Moissette, 75, pl. 11, fig. 8 .

1988 Hincksina flustroides (Hincks); Zabala et Maluquer, 80, figs. 86-87; pl. 3 , fig. A.

Tortonien supérieur: Fortuna. Pliocène: Los Terreros, Santa Pola.

\section{Hincksina loxopora (Reuss, 1848)}

1848 Cellepora loxopora Reuss, 97, pl. 11, fig. 24.

1992 Hincksina loxopora (R.); El Hajjaji, 91, pl. 4, fig. 5.
Tortonien: Rio Vinalopo. Tortonien supérieur: El Plomo. Pliocène: Santa Pola.

Genre Antropora Norman, 1903

Antropora lecointrei Buge, 1966

1966 Antropora lecointrei Buge, 25, fig. 2; pl. A, fig. 3. 1992 Antropora lecointrei Buge; El Hajjaji, 92, pl. 4, fig. 8.

Messinien: Carboneras.

Genre Aplousina Canu et Bassler, 1927

Aplousina bobiesi (David et Pouyet, 1974)

1974 Membranipora bobiesi David et Pouyet, 96, pl. 2, fig. 2.

1992 Aplousina bobiesi (D. et P.); El Hajjaji, 93, pl. 4, fig. 4. 1997b Aplousina bobiesi (D. et P.); Pouyet, 34, pl. 3, fig. 911.

Tortonien: Rio Vinalopo. Tortonien supérieur: Fortuna. Messinien: Sierra Alhamilla. Pliocène: Santa Pola.

Famille Calloporidae Norman, 1903

Genre Callopora Gray, 1848

Callopora fenestrata (Reuss, 1848)

1848 Cellepora fenestrata Reuss, 97, pl. 11, fig. 23.

1992 Callopora fenestrata (R.); El Hajjaji, 94, pl. 4, fig. 7.

1997b Callopora fenestrata (R.); Pouyet, 34, pl. 3, fig. 5, 6.

Tortonien supérieur: Fortuna. Pliocène: Santa Pola.

Genre Crassimarginatella Canu, 1900

Crassimarginatella crassimarginata (Hincks, 1880)

1880 Membranipora crassimarginata Hincks, 71, pl. 9, fig. 1.

1973 Crassimarginatella crassimarginata (H.); Harmelin, 483 , fig. $2 \mathrm{j} ; 3 \mathrm{e}, \mathrm{f}, \mathrm{g}$, i.

Un seul des 3 spécimens récoltés présente des aviculaires vicariants caractérisés par leur barre transversale servant de pivot à l'articulation de la mandibule. Harmelin note une différence dans la forme des aviculaires selon leur provenance: la mandibule est munie d'une pointe apicale chez les spécimens de Provence, tandis que les spécimens des Canaries et des Açores ont une mandibule arrondie. Harmelin et d'Hondt (1993) signalent cette espèce dans le benthos profond de la mer d'Alboran et notent qu'elle présente plutôt les caractéristiques méditerranéennes. Le matériel d'Espagne est de type nettement atlantique.

Messinien: Carboneras.

Cette espèce est bien connue en Méditerranée actuelle notamment en mer d'Alboran (Harmelin et d'Hondt, 1993) dans le domaine bathyal.

Genre Ramphonotus Norman, 1894 


\section{Ramphonotus minax (Busk, 1860)}

1860 Membranipora minax Busk, 125, pl. 25, fig. 1.

1995 Ramphonotus minax (Busk); Moissette et Spjeldnaes, 787, pl. 2, fig. 7.

1996 Ramphonotus minax (Busk); Haddadi-Hamdane, 60, pl. 4, fig. 1, 2.

Tortonien supérieur: El Plomo. Pliocène: Cuatro Calas, Los Terreros.

Famille Chaperiidae Jullien, 1888

Genre Chaperia Jullien, 1881

Chaperia annulus (Manzoni, 1870)

1870 Membranipora annulus Manzoni, 329, pl .1, fig. 6.

1975 Chaperia annulus (Manz.); Poluzzi, 47, pl. 21, fig. 5-6.

1988 Chaperia annulus (Manz.); Zabala et Maluquer, 86, fig. 107.

1992 Chaperia annulus (Manz.); El Hajjaji, 103, pl. 5, fig. 3.

1999 Chaperia annulus (Manz.); Pouyet et al., 126, fig. 3.1, 3.2.

Tortonien: Rio Vinalopo. Tortonien supérieur: Fortuna. Pliocène: Cuatro Calas, Los Terreros.

Famille Hiantoporidae MacGillivray, 1895

Genre Tremogasterina Canu, 1911

Tremogasterina bugei El Hajjaji, 1987

1987 Tremogasterina bugei El Hajjaji, 690, pl. 1, fig. 7-8.

1988 Tremogasterina sp. Moissette, 88, pl. 15, fig. 7, 10.

1992 Tremogasterina bugei El Hajj.; El Hajjaji, 155, pl. 8, fig. 6.

Cette espèce se reconnaît aisément par l'absence d'aviculaires.

Tortonien supérieur: Fortuna.

Tremogasterina cookae Pouyet et Moissette, 1986

Fig. $2 \mathrm{e}$

1986 Tremogasterina cookae Pouyet et Moissette, 386, pl. 1, fig. 9-10.

1992 Tremogasterina cookae P. et M.; El Hajjaji, 156, pl. 8, fig. 1.

Tortonien: Abanilla. Tortonien supérieur: El Plomo, Fortuna.

Genre Tremopora Ortmann, 1890

Tremopora radicifera (Hincks, 1881)

1881 Membranipora radicifera Hincks, 5, pl. 2, fig. 6.

1975 Tremopora radicifera (Hincks); Poluzzi, 48, pl. 19, fig. 8; pl. 20, fig. 7-9.

1992 Tremopora radicifera (Hincks); El Hajjaji, 104, pl. 5, fig. 1-2.

1997a Tremopora radicifera (Hincks); Pouyet, 15, fig. 6.
Tortonien: Abanilla. Tortonien supérieur: El Plomo, Fortuna, Sierra Gorda. Messinien: Carboneras. Pliocène: Cuatro Calas.

Famille Onychocellidae Jullien, 1881 Genre Onychocella Jullien, 1882

Onychocella angulosa (Reuss, 1848)

1848 Cellepora angulosa Reuss, 93, pl. 11, fig. 10.

1988 Onychocella angulosa (R.); Moissette, 91, pl. 1, fig. 45.

Comme nous l'avions déjà signalé (Pouyet, 1976), la distinction entre $O$. angulosa et $O$. marioni n'est pas aisée; en attendant des études plus poussées nous regroupons tous les spécimens sous le nom de $O$. angulosa.

Tortonien: Rio Vinalopo. Tortonien supérieur: Cabo Gata, El Plomo, Fortuna, Sierra Gorda. Messinien: Carboneras. Pliocène: Cuatro Calas, Los Terreros, Santa Pola.

Famille Microporidae Hincks, 1880

Genre Micropora Gray, 1848

Micropora coriacea (Johnston, 1847)

1847 Flustra coriacea Johnston, 349, pl. 56, fig. 8.

1988 Micropora coriacea (John.); Moissette, 93, pl. 14, fig. 9.

1988 Micropora coriacea (John.); Zabala et Maluquer, 90, fig. 125.

1992 Micropora coriacea (John.); Pouyet et Moissette, 40, pl. 4 , fig. 9.

Tortonien: Rio Vinalopo. Tortonien supérieur: El Plomo.

Micropora papyracea (Reuss, 1848)

1848 Cellepora papyracea Reuss, 94, pl. 11, fig. 15.

1992 Micropora papyracea (R.); El Hajjaji, 108, pl. 5, fig. 5.

Tortonien supérieur: Sierra Gorda.

Genre Mollia Lamouroux, 1821

Mollia patellaria (Moll, 1803)

1803 Eschara patellaria Moll, 68, pl. 4, fig. 20.

1992 Mollia patellaria (Moll); El Hajjaji, 109, pl. 5, fig. 12. 1997b Mollia patellaria (Moll); Pouyet, 40, pl. 3, fig. 7, 8.

Tortonien supérieur: Fortuna, Sierra Gorda.

Genre Aechmella Canu et Bassler, 1917 Aechmella poluzzii Moissette, 1988

1988 Aechmella poluzzii Moissette, 94, pl. 15, fig. 6, 9, 11. 1992 Aechmella poluzzii Mois.; El Hajjaji, 110, pl. 5, fig. 6.

Tortonien sunériar.. Fl Di^no. Messinien: Carboneras. 
Pliocène: Santa Pola.

Genre Steraechmella Lagaaij, 1952

Steraechmella buski Lagaaij, 1952

1952 Steraechmella buski Lagaaij, 39.

1992 Steraechmella buski Lag.; El Hajjaji, 112, pl. 6, fig. 1. 1997b Steraechmella buski Lag.; Pouyet, 41, pl. 4, fig. 4, 5.

Tortonien supérieur: Sierra Gorda. Pliocène: Cuatro Calas, Los Terreros.

Famille Calpensiidae Canu et Bassler, 1923

Genre Calpensia Jullien, 1888

Calpensia nobilis (Esper, 1796)

1796 Cellepora nobilis Esper, 145, pl. 7.

1988 Calpensia nobilis (Esper); Zabala et Maluquer, 90, fig. 126; pl. 2, fig. B.

1992 Calpensia nobilis (Esper); Pouyet et Moissette, 43, pl. 5, fig. 9.

1996 Calpensia nobilis (Esper); Haddadi-Hamdane, 67, pl. 5, fig. 10 .

Les spécimens du Pliocène inférieur de Santa Pola forment des nodules multilamellaires pouvant atteindre 7 à 8 centimètres de diamètre. Ce mode de croissance est fréquent chez cette espèce, le même phénomène se rencontre dans le Pliocène de Monastir (Tunisie).

Tortonien supérieur: El Plomo, Fortuna, Sierra Gorda. Pliocène: Santa Pola.

Famille Steginoporellidae Bassler, 1953

Genre Steginoporella Smitt, 1873

Steginoporella cucullata (Reuss, 1848)

Fig. 21

1848 Cellaria cucullata Reuss, 60, pl. 7, fig. 31.

1979 Steginoporella cucullata (R.); Pouyet et David, 774, fig. 3 ; pl. 3, fig. 10.

pars 1988 Steginoporella cucullata (R.); Moissette, 98, pl. 16, fig. 2-3.

Tortonien supérieur: El Plomo.

Steginoporella iberica iberica David et Pouyet, 1972

1972 Steginoporella iberica David et Pouyet, 239, pl. 14, fig. 1-6.

1979 Steginoporella iberica iberica D. et P.; Pouyet et David, 780, fig. 3; pl. 2, fig. 7.

1992 Steginoporella iberica iberica D. et P.; El Hajjaji, 120, pl. 6 , fig. 5 .

Tortonien supérieur: El Plomo, Fortuna. Messinien: Sierra Alhamilla.
1972 Steginoporella montenati David et Pouyet, 240, pl. 15, fig. 1-6; pl. 16, fig. 1-6.

1979 Steginoporella montenati D. et P.; Pouyet et David, 787, fig. 3; pl. 2, fig. 8.

1992 Steginoporella montenati D. et P.; El Hajjaji, 122, pl. 6, fig. 11.

1993 Steginoporella montenati D. et P.; Moissette et al., 96, fig. 5 e-h.

Tortonien: Rio Vinalopo. Pliocène: Santa Pola.

Famille Aspidostomatidae Jullien , 1888

Genre Monoporella Hincks, 1881

Monoporella venusta (Eichwald, 1853)

Fig. 2 h - i

1853 Cellepora venusta Eichwald, 29, pl. 2, fig. 2.

1992 Monoporella venusta (Eich.); El Hajjaji, 123, pl. 5, fig. 15.

Messinien: Carboneras.

Famille Setosellidae Levinsen, 1909

Genre Setosella Hincks, 1877

Setosella vulnerata (Busk, 1860)

1860 Membranipora vulnerata Busk, 124, pl. 25, fig. 3.

1988 Setosella vulnerata (Busk); Zabala et Maluquer, 93, fig. 133, pl. 2, fig. E.

1992 Setosella vulnerata (Busk); Reguant et Maluquer, 144, pl. 1 , fig. 6.

1993 Setosella vulnerata (Busk); Moissette et al., 97, fig. 6a-b.

1995 Setosella vulnerata (Busk); Moissette et Spjeldnaes, 786, pl. 2, fig. 8.

Messinien: Carboneras.

Famille Cellariidae Hincks, 1880

Genre Cellaria Ellis et Solander, 1786

Cellaria fistulosa Linné, 1758

1758 Cellaria fistulosa Linné, 804.

1992 Cellaria fistulosa Auct.; Pouyet et Moissette, 46, pl. 5, fig. 11-12.

1992 Cellaria fistulosa Auct.; El Hajjaji, 126, pl. 6, fig. 4. 1999 Cellaria fistulosa L.; Pouyet et al., 131, fig. 5.3.

Messinien: Sierra Alhamilla. Pliocène: Cuatro Calas, Los Terreros, Santa Pola.

\section{Cellaria melillensis El Hajjaji, 1987}

1987 Cellaria melillensis El Hajjaji; 690, pl. 1, fig. 2-3.

1988 Cellaria melillensis El Hajj.; Moissette, 105, pl. 3, fig. 2; pl. 17, fig. 6-7.

1992 Cellaria melillensis El Hajj.; El Hajjaji, 127, pl. 6, fig. 2.

Messinien: Carboneras. 
Famille Scrupocellariidae Levinsen, 1909

Genre Scrupocellaria Van Beneden, 1845

Scrupocellaria elliptica (Reuss, 1848)

1848 Bactridium ellipticum Reuss, 56, pl. 9, fig. 7-8.

1989 Scrupocellaria elliptica (R.); Schmid, p. 23, taf. 5, fig. $1-7$.

1992 Scrupocellaria elliptica (R.); El Hajjaji, p. 131, pl. 6, fig. 8.

1996 Scrupocellaria elliptica (R.); Haddadi-Hamdane, 73, pl. 5 , fig. 5 .

Pliocène: Cuatro Calas.

SOUS-ORDRE ASCOPHORA Levinsen, 1909

Famille Cribrilinidae Hincks, 1880

Genre Cribrilina Gray, 1848

Cribrilina messiniensis Pouyet et Moissette, 1986

1986 Cribrilina messiniensis Pouyet et Moissette, 387, pl. 1, fig. 7-8.

1992 Cribrilina messiniensis P. et M.; El Hajjaji, 134, pl. 6, fig. 17.

Tortonien supérieur: Fortuna, Sierra Gorda. Messinien: Carboneras.

Genre Figularia Jullien, 1886

Figularia figularis (Johnston, 1847)

1847 Lepralia figularis Johnston, 314, pl. 56, fig. 2.

1976 Figularia manzonii (Reuss); Pouyet, 62.

1988 Figularia figularis (John.); Zabala et Maluquer, 110, fig. 222, pl. 7, fig. H.

1992 Figularia figularis (John.); Pouyet et Moissette, 49, pl. 6 , fig. 8

1996 Figularia figularis (John.); Haddadi-Hamdane, 77, pl. 6 , fig. 1.

En 1976, nous avions attribué le matériel pliocène de Cuatro Calas à $F$. manzonii en nous basant sur les plus grandes dimensions de notre matériel tout en notant les difficultés à séparer des espèces uniquement sur des caractères dimensionnels.

Tortonien supérieur: Cabo Gata, El Plomo, Fortuna, Sierra de Crevillente, Sierra Gorda. Pliocène: Cuatro Calas, Santa Pola.

\section{Genre Gephyrotes Norman, 1903 \\ Gephyrotes fortunensis n. sp.}

Fig. 3 i -k

1988 Gephyrotes sp. Moissette, 112, pl. 18, fig. 8, 11.

Holotype: spécimen figuré FSL 117925.

Locus typicus: Fortuna (Murcie, Espagne), au nord du village sur la route nouvelle à $800 \mathrm{~m}$ avant l'embranchement en direction de Barinas.

Stratum typicum: Tortonien supérieur.

Derivatio nominis: du nom du gisement type.

Diagnosis: Gephyrotes caractérisé par 4 à 5 paires de costules latérales et une ovicelle avec fenêtres et une crête médiane.

Gephyrotes with 4 or 5 lateral costulae and with an ovicell with 2 lateral fenestrae and a median crest.

\section{Description}

Zoarium encroûtant. Zoécies distinctes, ovales, bombées. Frontale formée de 8 à 10 costules séparées par de larges lacunes. Bouclier central garni de pores. Les deux premières paires de côtes peuvent se développer jusqu'à former une barre au-dessus de l'aperture avec grosse lacune médiane. Aperture semi-circulaire avec deux épines distales. De chaque côté de l'orifice, il peut y avoir un grand aviculaire, courbe, orienté distalement. Ovicelle hyperstomiale, globuleuse, avec 2 fenêtres de chaque côté d'une crête médiane saillante. Pas d'aviculaires interzoéciaux.

Dimensions de l'holotype:

Longueur zoéciale: 0,52 - 0,68

Largeur zoéciale: $0,35-0,41$

Hauteur aperture: $0,10-0,13$

Largeur aperture: $0,15-0,20$

Matériel: une douzaine de spécimens tous en provenance du gisement de Fortuna.

\section{Remarque}

La comparaison avec le matériel du Messinien d'Algérie (Moissette, 1988) a permis de montrer l'identité des 2 formes.

La distribution stratigraphique du genre Gephyrotes semble être limitée au Tertiaire ancien d'Amérique du

Figure 2. a - b. Microporella coronata (Savigny-Audouin), Pliocène inf., Santa Pola. FSL 117 333. a x 48 , b X 80. c. Cryptosula pallasiana (Moll), Tortonien sup., Sierra Gorda. FSL 493 174. x 40. d. Hippomenella mucronelliformis (Waters), Pliocène inf., Santa Pola. FSL 117 301. x 40. e. Tremogasterina cookae Pouyet et Moissette, Tortonien sup., Fortuna. FSL 117 545. x 40. f. Microporella ciliata (Pallas), Tortonien sup., El Plomo, FSL 493 173. x 52. g. Hincksina flustroides (Hincks), Pliocène inf., Santa Pola. FSL 117 333. x 40. h - i. Monoporella venusta (Eichwald), Messinien, Carboneras. FSL 117 851. h x 40, i x 28. j. Schizoporella geminipora (Reuss), Tortonien sup., El Plomo, FSL 493 175. x 60. k. Hippomenella mucronelliformis (Waters), Pliocène inf., Santa Pola. FSL 117 301. x 62. 1. Steginoporella cucullata (Reuss), Tortonien sup., El Plomo, FSL 493 165. x 24. m. Monoporella venusta (Eichwald), Messinien, Carboneras. FSL 117 851. x 120. 

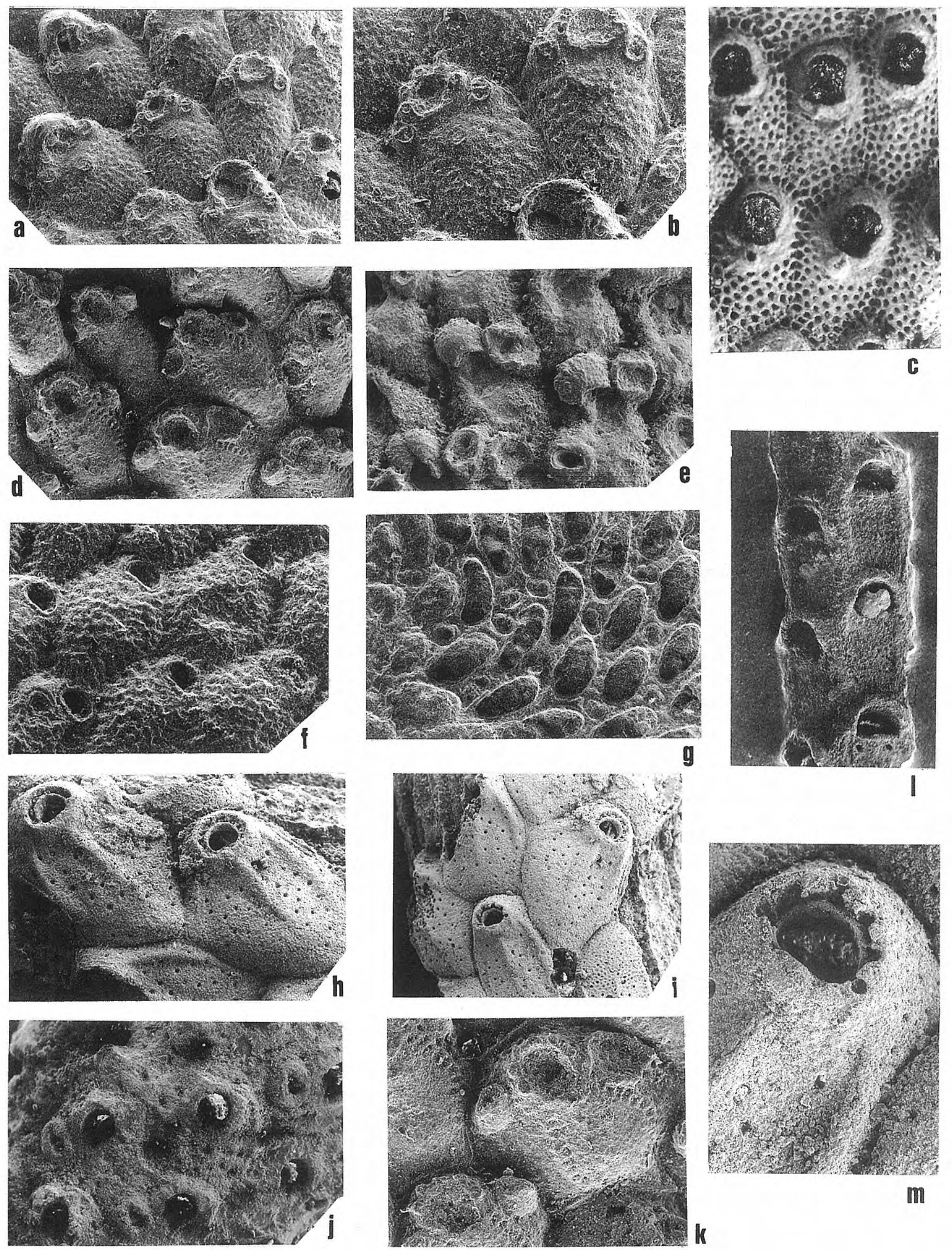
Nord. Dans les mers actuelles Gephyrotes n'a été cité que dans les zones boréales atlantiques. Gephyrotes nitidopunctata (Smitt) a plus de costules, son ovicelle est lisse et l'aviculaire latéral à l'aperture est dirigé proximalement.

Aucune forme fossile n'a été décrite dans le Tertiaire ancien ou récent du domaine méditerranéen ou atlantique nord-oriental; une comparaison avec les formes paléogènes du continent nord-américain ne nous semble pas utile.

Genre Membraniporella Smitt, 1873

Membraniporella nitida (Johnston, 1838)

1838 Lepralia nitida Johnston, 277, pl. 34, fig. 7.

1988 Membraniporella nitida (John.); Zabala et Maluquer, 111, fig. 223; pl. 8, fig. A.

1992 Membraniporella nitida (John.); Pouyet et Moissette, 49, pl. 6, fig. 10.

Cette espèce est très rare dans nos gisements.

Tortonien supérieur: Fortuna. Pliocène: Cuatro Calas.

Genre Puellina Jullien, 1886

Puellina (Cribrilaria) cf. bifida (d'Hondt, 1970)

Un seul spécimen a été attribué avec doute à cette espèce. Les zoécies présentent 6 à 11 costules, saillantes, aigües. Les zoécies non ovicellées ont 5 épines péristomiales, celles qui sont ovicellées seulement 4 . Présence d'aviculaires interzoéciaux à base élargie et à mandibule triangulaire. Ovicelle avec protubérances.

Remarque: Bishop et Househam (1987) rapprochent cette espèce de P. kollmanni (David et Pouyet, 1974) du Badénien d'Eisenstadt. Il se pourrait que l'espèce décrite par El Hajjaji (1992) sous le nom de P. kollmanni soit en réalité $P$. bifida.

Messinien: Carboneras.

Puellina (Cribrilaria) innominata (Couch, 1844)

Fig. $3 \mathrm{~d}-\mathrm{e}$

1844 Lepralia innominata Couch, 114, pl. 22, fig. 4.

1986 Cribrilaria innominata (Couch); Bishop, 96-98, figs. 1-8.

1987 Puellina (Crib.) innominata (Couch); Bishop et Househam, 33-37, figs. 50-58.

1988 Puellina (Crib.) innominata (Couch); Zabala et Maluquer, 108, fig. 220, pl. 6, fig. 6 E-F.

Tortonien: Rio Vinalopo. Tortonien supérieur: El Plomo, Fortuna, Sierra Gorda. Messinien: Carboneras. Pliocène: Santa Pola.

Puellina (Cribrilaria) radiata (Moll, 1803)
1970 Puellina radiata (Moll); Harmelin, 80, fig. 1a-c, 3a; pl. 1, fig. 1-3.

1988 Puellina (Crib.) radiata Moll; Zabala et Maluquer, 107, fig. 214; pl. 7, fig. A-B.

Tortonien: Abanilla. Tortonien supérieur: Fortuna. Pliocène: Cuatro Calas, Santa Pola.

Puellina (Cribrilaria) scripta (Reuss, 1848)

1848 Cellepora scripta Reuss, 82, pl. 9, fig. 28.

1988 Puellina (Crib.) scripta (R.); Harmelin et Aristegui, 526, figs. 18-20, 24.

1988 Puellina (Crib.) scripta (R.); Zabala et Maluquer, 108, fig. 221

1992 Puellina (Crib.) scripta (R.); El Hajjaji, 138, pl. 7, fig. 1.

1993 Puellina scripta (R.); Harmelin et d'Hondt, 67, fig. 5. 1997b Puellina scripta (R.); Pouyet, 46, pl. 4, fig. 7-9.

Certains auteurs mettent en synonymie $P$. radiata et P. scripta. Les travaux de Harmelin (1970), Harmelin et Aristegui (1988), et Harmelin et d'Hondt (1993) démontrent cependant que ces deux espèces sont bien distinctes.

Tortonien supérieur: El Plomo, Fortuna. Pliocène: Santa Pola.

Puellina (Glabrilaria) pedunculata Gautier, 1956

1956 Puellina pedunculata Gautier, 203, fig. 20.

1987 Puellina (Glab.) pedunculata Gautier; Bishop et Househam, 55-59, figs. 95-97.

1988 Puellina (Glab.) pedunculata Gautier; Harmelin, 31, fig. 10, 11, 16, 17 a-c.

1988 Puellina (Glab.) pedunculata Gautier; Zabala et Maluquer, 107, fig. 209; pl. 7, fig. E.

1992 Puellina (Glab.) pedunculata Gautier; El Hajjaji, 140, pl. 7, fig. 2-3.

1993 Puellina (Glab.) pedunculata Gautier; Harmelin et d'Hondt, fig. 9.

Un seul spécimen asez mal conservé. Petite taille des zoécies ayant 11 à 13 costules. Orifice semi-circulaire. Six épines orales. Ovicelle carénée. Aviculaires pédonculés, très rares, entre les zoécies. Une seule zoécie avortée sur notre zoarium.

Messinien: Carboneras.

Puellina (Glabrilaria) orientalis Harmelin, 1988

1988 Puellina (Glabrilaria) orientalis Harmelin, 31, fig. 12$13,17 \mathrm{j}, \mathrm{k}, \mathrm{l}$.

1988 Puellina (Glab.) orientalis Harmelin ssp orientalis; Zabala et Maluquer, 107, fig. 208.

Deux spécimens de très petite taille et mal préservés. Cette espèce se différencie de la précédente par un 
bouclier frontal plus aplati, des aviculaires non pédonculés accolés à l'ovicelle.

Tortonien supérieur: Fortuna.

Famille Hippothoidae Levinsen, 1909

Genre Chorizopora Hincks, 1880

Chorizopora brongniarti (Savigny-Audouin, 1828)

1828 Flustra Brongniarti Savigny Audouin, 68, pl. 10, fig. 6.

1992 Chorizopora brongniarti (Savi.-Aud.); El Hajjaji, 157, pl. 8 , fig. 5 .

1992 Chorizopora brongniarti (Savi.-Aud.); Pouyet et Moissette, 50, pl. 7, fig. 1.

Tortonien supérieur: Fortuna, Sierra Gorda. Messinien: Carboneras. Pliocène: Cuatro Calas, Los Terreros, Santa Pola.

Genre Trypostega Levinsen, 1909

Trypostega venusta (Norman, 1864)

1864 Lepralia venusta Norman, 84, pl. 10, fig. 2, 3.

1988 Trypostega venusta (Nor.); Moissette, 115, pl. 19, fig. $1,2$.

1988 Trypostega venusta (Nor.); Zabala et Maluquer, 144, fig. 357, pl. 19, fig. E.

Tortonien: Abanilla, Campules.

Famille Umbonulidae Canu, 1904

Genre Umbonula Hincks, 1880

Umbonula macrocheila (Reuss, 1848)

1848 Eschara macrocheila Reuss, 65, pl. 8, fig. 14.

1997b Umbonula macrocheila (R.); Pouyet, 50, pl. 4, fig. 10, pl. 5 , fig. 5 .

Tortonien: Rio Vinalopo. Tortonien supérieur: El Plomo.

Umbonula monoceros (Reuss, 1848)

1848 Cellepora monoceros Reuss, 80, pl. 9, fig. 24.

1992 Umbonula monoceros (R.); El Hajjaji, 146, pl. 7, fig. 11.

Messinien: Carboneras.

Genre Hippopleurifera Canu, 1927

Hippopleurifera benmoussai El Hajjaji, 1992

1992 Hippopleurifera benmoussai El Hajjaji, 147, pl. 7, fig. 12-13.

Messinien: Carboneras.

Hippopleurifera sedgwicki (Milne Edwards, 1836)
1836 Eschara Sedgwicki Milne Edwards, 330, pl. 10, fig. 5. 1992 Hippopleurifera sedgwicki (M. Edw.); El Hajjaji, 148, pl. 8 , fig. 3 .

1997a Hippopleurifera sedgwicki (M. Edw.); Pouyet, 25, text-fig. 17.

Pliocène: Cuatro Calas, Los Terreros.

$$
\text { Hippopleurifera surgens (Manzoni, 1875) }
$$

1875 Lepralia surgens Manzoni, 18, pl. 2, fig. 22.

1976 Hippopleurifera surgens (Manz.); Pouyet, 63, pl. 10, fig. 1.

1992 Hippopleurifera surgens (Manz.); Pouyet et Moissette, 52 , pl. 7, fig. 4, 7.

1996 Hippopleurifera surgens (Manz.); Haddadi-Hamdane, 79 , pl. 6, fig. 5 .

Tortonien: Rio Vinalopo. Tortonien supérieur: Fortuna, Sierra Gorda. Pliocène: Cuatro Calas, Los Terreros, Santa Pola.

Famille Schizoporellidae Jullien, 1903 Genre Schizoporella Hincks, 1877

Schizoporella geminipora (Reuss, 1848) Fig. 2 j

1848 Vaginopora geminipora Reuss, 74, pl. 9, fig. 3-4. 1992 Schizoporella geminipora (R.); El Hajjaji, 193, pl. 12, fig. 2-3.

Très rare dans deux gisements.

Tortonien supérieur: El Plomo. Messinien: Sierra Alhamilla.

\section{Schizoporella longirostris Hincks, 1886}

1886 Schizoporella unicornis forma longirostris Hincks, 226, pl. 10, fig. 2.

1988 Schizoporella longirostris H.; Zabala et Maluquer, 133, fig. 312; pl. 18, fig. C-D.

1992 Schizoporella longirostris H.; El Hajjaji, 195, pl. 12, fig. 5 .

1992 Schizoporella longirostris H.; Pouyet et Moissette, 54, pl. 7 , fig. 11.

1995 Schizoporella dunkeri (Reuss); Hayward et Ryland, 39, pl. 2, 3 .

Tortonien: Rio Vinalopo. Tortonien supérieur: Fortuna, Sierra Gorda.

Schizoporella tetragona (Reuss, 1848)

1848 Cellepora tetragona Reuss, 78, pl. 9, fig. 19.

1974 Schizoporella tetragona (R.); David et Pouyet, 156, pl. 15 , fig. 1.

1989 Schizoporella tetragona (R.); Schmid, 43, pl. 12, fig. 8. 
Tortonien supérieur: Sierra Gorda.

Schizoporella unicornis (Johnston, 1847)

1847 Lepralia unicornis Johnston, 320, pl. 57, fig. 1. 1988 Schizoporella unicornis (John.); Moissette, 127.

1988 Schizoporella unicornis (John.); Zabala et Maluquer, 133, fig. 309.

Tortonien supérieur: Sierra Gorda. Pliocène: Santa Pola.

Genre Cribellopora Gautier, 1957

Cribellopora latigastra (David, 1949)

1949 Arthropoma latigastra David, 542, pl. 20, fig. 1, 5.

1992 Cribellopora latigastra (David); El Hajjaji, 197, pl. 10, fig. 12 .

Tortonien supérieur: Fortuna, Sierra Gorda.

Genre Emballotheca Levinsen, 1909

Emballotheca longidens (Cipolla, 1921)

1921 Hippoporina longidens Cipolla, 96, pl. 4, fig.17-18.

1986 Emballotheca mediterranea Pouyet et Moissette, 388, pl. 1, fig. 1-4.

1992 Emballotheca longidens (Cip.); Pouyet et Moissette, 55, pl. 8, fig. 5-6.

1996 Emballotheca longidens (Cip.); Haddadi-Hamdane, 88, pl. 7 , fig. 5 .

1997b Emballotheca longidens (Cip.); Pouyet, 56, pl. 5, fig. $8,9$.

Tortonien supérieur: Fortuna. Pliocène: Los Terreros, Santa Pola.

Genre Escharina Milne Edwards, 1836

Escharina dutertrei (Savigny-Audouin, 1828)

1828 Flustra Dutertrei Savigny Audouin, 239, pl. 9, fig. 2. 1976 Schizoporella sp. C, Pouyet, 65, pl. 14, fig. 3.

1988 Escharina dutertrei (Sav.-Aud.); Zabala et Maluquer, 128, figs. 293, 293 bis, pl. 15, fig. A-C.

1992 Escharina dutertrei (Sav.-Aud.); Pouyet et Moissette, 56, pl. 8, fig. 1 .

1996 Escharina dutertrei (Sav.-Aud.); Haddadi-Hamdane, 82 , pl. 6 , fig. 7 .

Cette espèce avait été décrite et figurée en 1976 (Pouyet, p. 65) sous le nom de Schizoporella sp $C$.

Messinien: Carboneras. Pliocène: Cuatro Calas, Los
Terreros, Santa Pola.

$$
\text { Escharina vulgaris (Moll, 1803) }
$$

1803 Eschara vulgaris Moll, 55, pl. 3, fig. 10-11.

1992 Escharina vulgaris (Moll); El Hajjaji, 201, pl. 12, fig. 11.

1992 Escharina vulgaris (Moll); Pouyet et Moissette, 56, pl. 8, fig. 2.

Tortonien: Rio Vinalopo. Tortonien supérieur: Fortuna. Pliocène: Cuatro Calas, Los Terreros, Santa Pola.

Genre Herentia Gray, 1848

Herentia (Herentia) hyndmanni (Johnston, 1847)

1847 Lepralia Hyndmanni Johnston, 306, pl. 54, fig. 6. 1992 Herentia (Herentia) hyndmanni (John.); Pouyet et Moissette, 57, pl. 8, fig. 3.

1996 Herentia (Herentia) hyndmanni (John.); HaddadiHamdane, 83, pl. 6, fig. 10.

Messinien: Carboneras.

$$
\text { Herentia (Therenia) milneana (Busk, 1859) }
$$

1859 Lepralia milneana Busk, 132.

1989 Herentia milneana (Busk); Bishop et Hayward, 20, figs. 79-82, 248.

1992 Herentia (Therenia) milneana (Busk); El Hajjaji, 204, pl. 11, fig. 3 .

Tortonien: Rio Vinalopo.

Herentia (Therenia) montenati Pouyet, 1976

1976 Herentia montenati Pouyet, 66, pl. 12, fig. 5.

1992 Herentia (Therenia) montenati P.; El Hajjaji, 205, pl. 11 , fig. 7.

1996 Herentia (Therenia) montenati P.; Haddadi-Hamdane, 84 , pl. 6, fig. 3 .

Tortonien: Rio Vinalopo.Tortonien supérieur: El Plomo, Fortuna. Pliocène: Cuatro Calas, Los Terreros, Santa Pola.

Herentia (Herentia) thalassae alboransis Pouyet et Moissette, 1986

1986 Herentia thalassae alboransis Pouyet et Moissette, 389, pl. 1, fig. 5-6.

1992 Herentia (Herentia) thalassae alboransis P. et M.; El Hajjaji, 206, pl. 11, fig. 5.

Figure 3. a. Adeonellopsis distoma (Busk), Messinien, Carboneras. FSL 492 141. x 62. b. Rhynchozoon digitatum (Waters), Messinien, Carboneras. FSL 493 182. x 32. c. Hippomonavella moissettei El Hajjaji, Tortonien sup., Fortuna. FSL 117 993. x 80. d - e. Puellina (Cribrilaria) innominata (Couch), Tortonien sup., Fortuna. FSL 492 049. d x 54, e x 100. f. Hippomonavella moissettei El Hajjaji, Tortonien sup., Fortuna. FSL 117 993. x 52. g - h. Metrarabdotos canui Buge et Galopim de Carvalho, Tortonien sup., El Plomo. FSL 493 147. g x 54. h, détail de l'ovicelle, x 66. i k. Gephyrotes fortunensis n. sp., Tortonien sup., Fortuna. FSL 117 925. i x 66, j x 100, k x 48. 

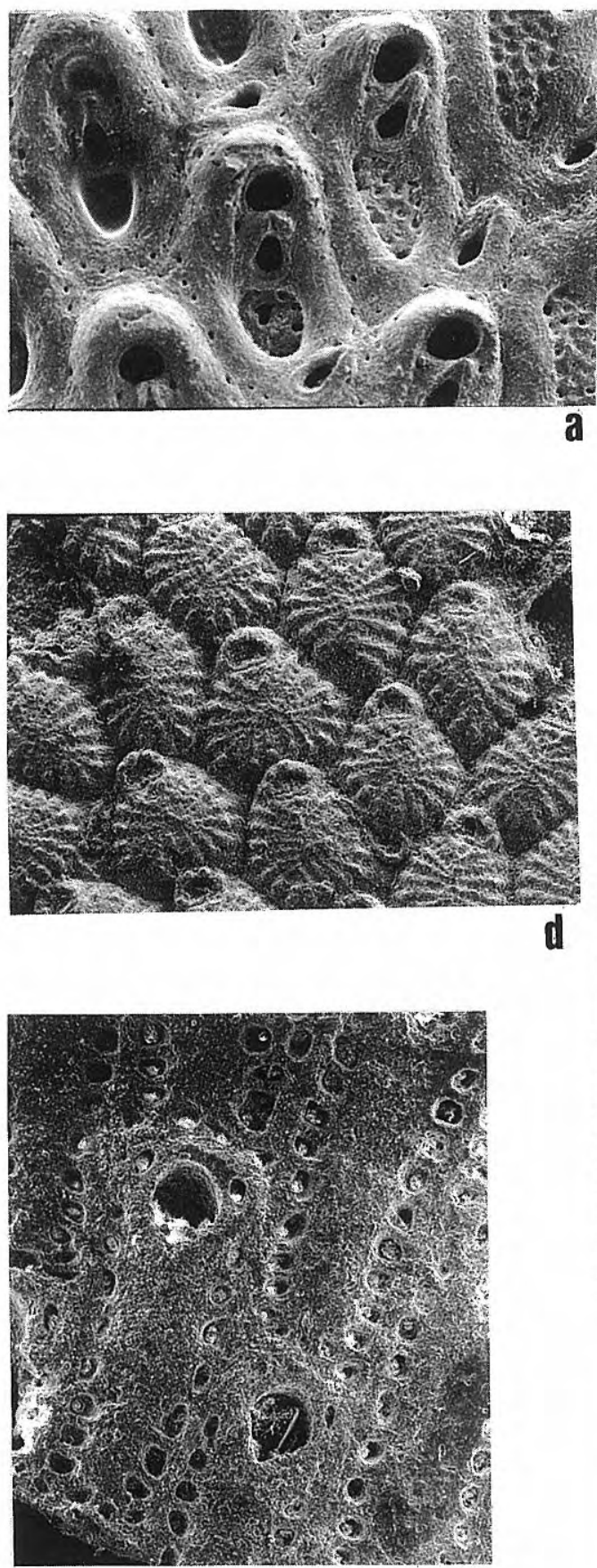

g

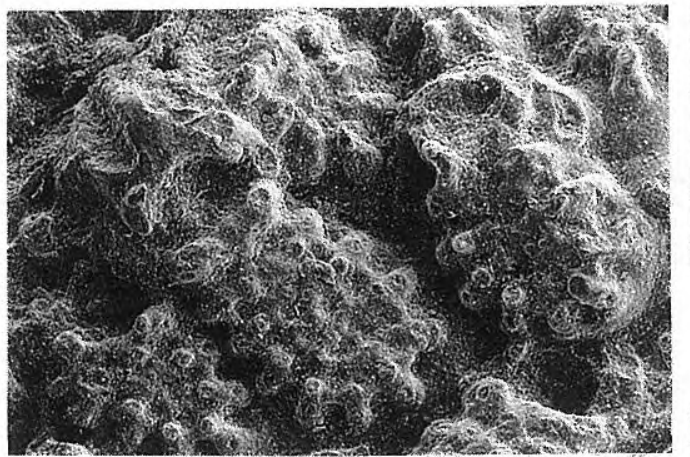

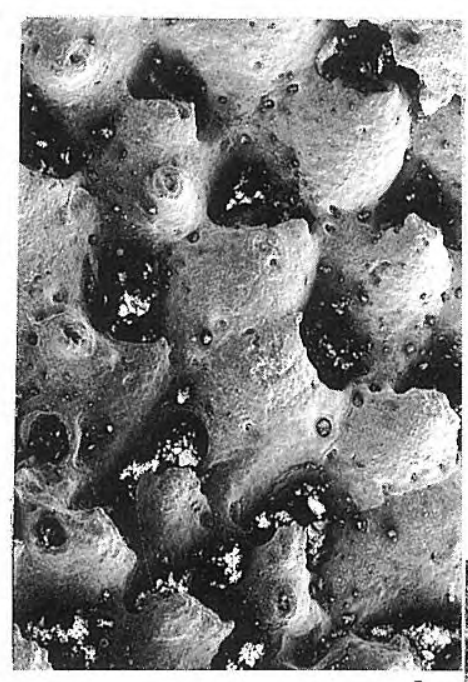

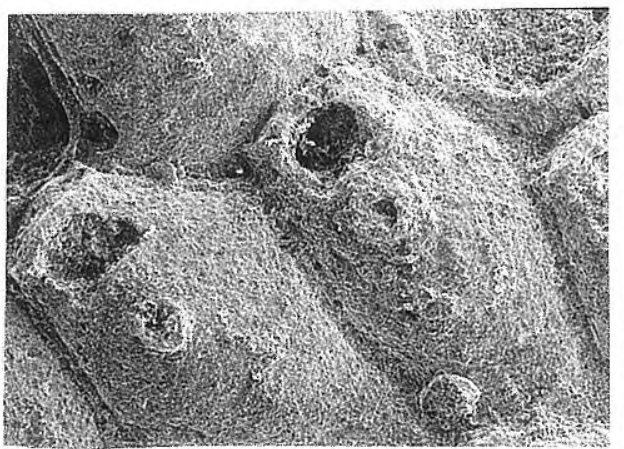

C

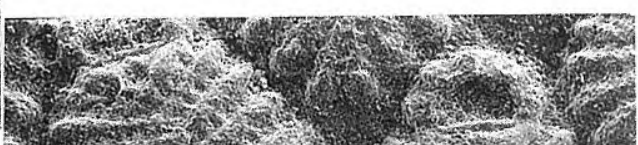

b
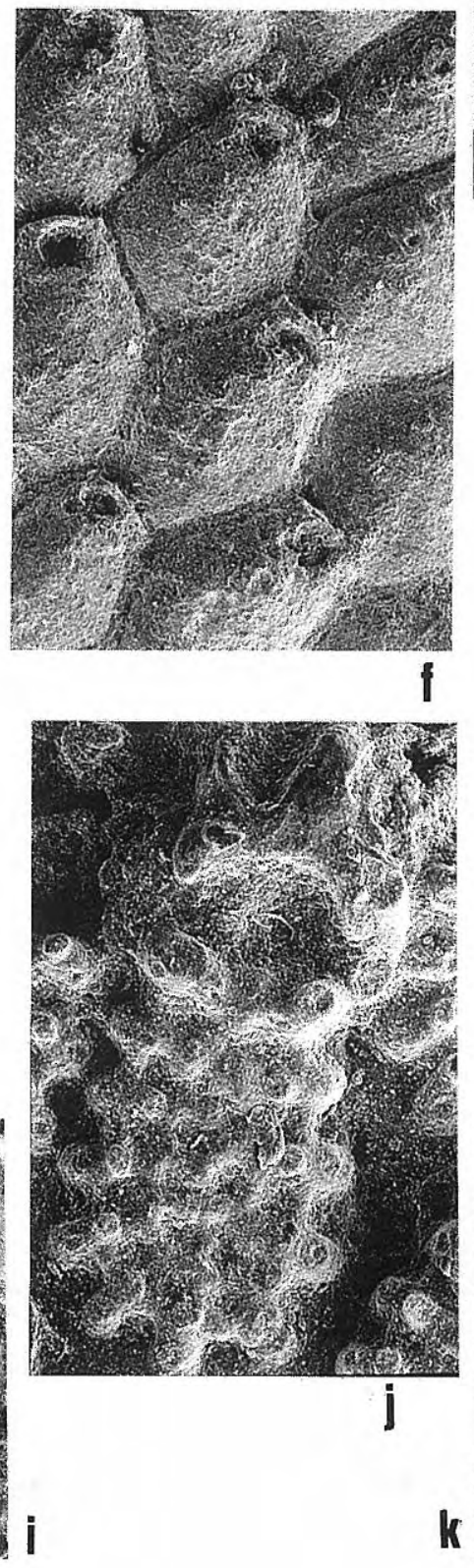

F 1. $x$.

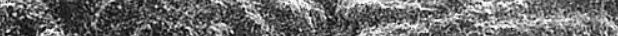

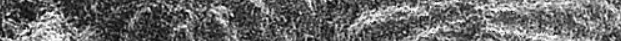
T. Mr.t.

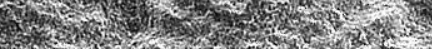

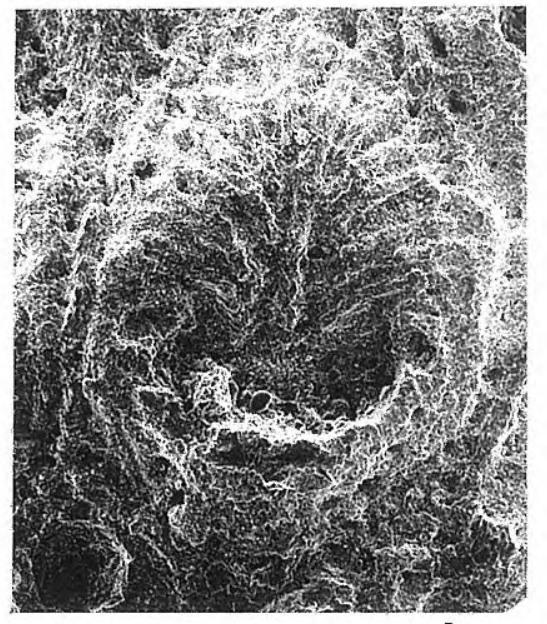

h

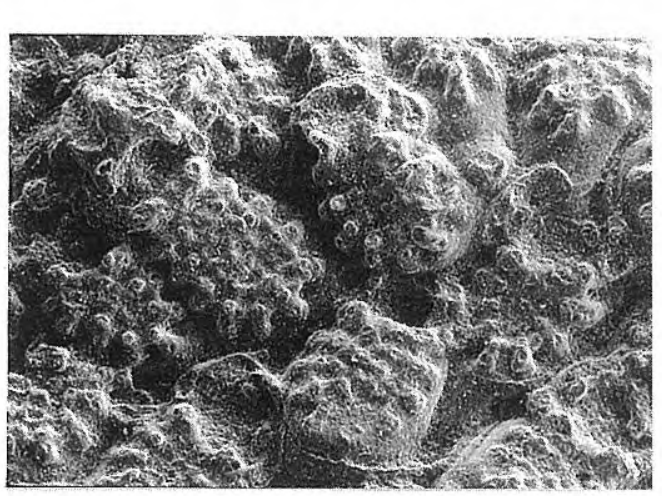


Tortonien supérieur: Fortuna.

Genre Schizobrachiella Canu and Bassler, 1920 Schizobrachiella sanguinea (Norman, 1868)

1868 Hemeschara sanguinea Norman, 222, pl. 7, fig. 9-11. 1988 Schizobrachiella sanguinea (Norm.); Zabala et Maluquer, 130, fig. 295, pl. 16, fig. E.

1992 Schizobrachiella sanguinea (Norm.); El Hajjaji, 209, pl. 12 , fig. 9 .

1992 Schizobrachiella sanguinea (Norm.); Pouyet et Moissette, 58, pl. 8, fig. 8.

Tortonien supérieur: Sierra Gorda. Pliocène: Santa Pola.

Genre Schizomavella Canu et Bassler, 1917

Schizomavella auriculata (Hassall, 1842)

1842 Lepralia auriculata Hassall, 412.

1988 Schizomavella auriculata (Hass.); Zabala et Maluquer, 132, fig. 301, pl. 17, fig. A.

1992 Schizomavella auriculata (Hass.); El Hajjaji, 210, pl. 11 , fig. $1,4$.

1992 Schizomavella auriculata (Hass.); Pouyet et Moissette, 58 , pl. 8 , fig. 11 .

Tortonien: Rio Vinalopo. Tortonien supérieur: Fortuna, Sierra Gorda. Pliocène: Los Terreros.

\section{Schizomavella linearis (Hassall, 1842)}

1842 Lepralia linearis Hassall, 368, pl. 9, fig. 8.

1988 Schizomavella linearis (Hass.); Zabala et Maluquer, 132, fig. 300, pl. 19, fig. A.

1992 Schizomavella linearis (Hass.); Pouyet et Moissette, 59, pl. 8, fig. 9, 12 .

Tortonien supérieur: El Plomo. Pliocène: Cuatro Calas, Los Terreros.

Genre Schismoporella Gregory, 1893

Schismoporella aculifera (Canu et Lecointre, 1925)

1925 Schizomavella aculifera Canu et Lecointre, 76, pl. 10, fig. 8-10.

1992 Schismoporella aculifera (C. et L.); El Hajjaji, 207, pl. 12, fig. 8.

Tortonien supérieur: Fortuna. Pliocène: Santa Pola.

Famille Hipporinidae Bassler, 1935

Genre Hippoporina Neviani, 1895

Hippoporina canui Cipolla, 1921

1921 Hippoporina Cánui Cipolla, 253, pl. 4, fig. 15-16.

1992 Hippoporina canui Cip.; El Hajjaji, 163, pl. 8, fig. 13.

1992 Hippoporina canui Cip.; Pouyet et Moissette, 60, pl. 8, fig. 10.

Tortonien supérieur: El Plomo, Fortuna, Sierra Gorda.
Pliocène: Los Terreros.

Hippoporina odontostoma (Reuss, 1874)

1874 Lepralia odontostoma Reuss, 156, pl. 4, fig. 8.

1997b Hippoporina odontostoma (R.); Pouyet, 61, pl. 6, fig. $2-4$.

Tortonien supérieur: Fortuna. Pliocène: Santa Pola.

Genre Buffonellodes Strand, 1928

Buffonellodes incisa (Reuss, 1874)

1874 Lepralia incisa Reuss, 168, pl. 3, fig.4.

1992 Buffonellodes incisa (R.); El Hajjaji, 213, pl. 11, fig. 10.

1992 Buffonellodes incisa (R.); Pouyet et Moissette, 61, pl. 9 , fig. 3.

1997b Buffonellodes incisa (R.); Pouyet, 62, pl. 6, fig. 5.

Tortonien supérieur: El Plomo, Fortuna, Sierra Gorda. Pliocène: Cuatro Calas, Los Terreros, Santa Pola.

Genre Hippoporella Canu, 1917

Hippoporella pauper (Reuss, 1874)

1874 Lepralia pauper Reuss, 164, pl. 5, fig. 4.

1988 Hippoporella pauper (R.); Moissette, 146, pl. 23, fig. 9, 12.

1989 Hippoporella pauper (R.); Schmid, 48, pl. 14, fig. 1-3.

Tortonien supérieur: Fortuna.

Genre Hippomenella Canu et Bassler, 1917

Hippomenella mucronelliformis (Waters, 1899)

Fig. $3 \mathrm{~d}, \mathrm{k}$

1899 Lepralia mucronelliformis Waters, 11, pl. 3, fig. 21.

1988 Hippomenella mucronelliformis (W.); Zabala et Maluquer, 117, fig. 244, pl. $8 \mathrm{H}$.

1996 Hippomenella mucronelliformis (W.); HaddadiHamdane, 90, pl. 7, fig. 1.

Tortonien: Rio Vinalopo. Pliocène: Santa Pola.

En Méditerranée orientale, cette espèce vit entre 100 et $200 \mathrm{~m}$ de profondeur.

Genre Stephanosella Canu et Bassler, 1917

Stephanosella entomostoma (Reuss, 1848)

1848 Cellepora entomostoma Reuss, 92, pl. 11, fig. 7.

1992 Stephanosella entomostoma (R.); El Hajjaji, 214, pl. 11, fig. 13.

1993 Stephanosella entomostoma (R.); Moissette et al., 103, text-fig. $6 \mathrm{~h}-\mathrm{i}$.

Tortonien supérieur: El Plomo, Fortuna, Sierra Gorda. 
Genre Hippodiplosia Canu, 1916

Hippodiplosia miocenica Moissette, 1988

1988 Hippodiplosia miocenica Moissette, 135, pl. 21, fig. 11; pl. 22, fig.1.

1992 Hippodiplosia miocenica Mois.; El Hajjaji, 167, pl. 9, fig. 5.

Tortonien supérieur: Fortuna. Pliocène: Santa Pola.

Hippodiplosia ottomulleriana (Moll, 1803)

1803 Eschara otto-mulleriana Moll, 60, pl. 3, fig. 15 A-C.

Tortonien: Rio Vinalopo.

Genre Hippomonavella Bassler, 1934

Hippomonavella moissettei El Hajjaji, 1992

Fig. 3 c, f

1992 Hippomonavella moissettei El Hajjaji, 169, pl. 9, fig. 7-8.

Tortonien supérieur: Fortuna, Sierrra Gorda.

Famille Exochellidae Bassler, 1935

Genre Escharoides Milne Edwards, 1836

Escharoides coccinea (Abildgaard, 1806)

1806 Cellepora coccinea Abildgaard, 30, pl. 116, fig. 1-2.

1988 Escharoides coccinea (Abild.); Moissette, 148, pl. 24, fig. 4.

Tortonien: Campules, Rio Vinalopo. Tortonien supérieur: El Plomo, Fortuna, Sierra Gorda. Pliocène: Cuatro Calas, Los Terreros, Santa Pola.

\section{Escharoides fulgurans (Manzoni, 1869)}

1869 Lepralia fulgurans Manzoni, 936, pl. 1, fig.6.

1976 Escharoides fulgurans (Manz.); Pouyet, 68, pl. 11, fig. 5.

1988 Escharoides fulgurans (Manz.); Moissette, 149, pl. 24, fig. 11.

1992 Escharoides fulgurans (Manz.); Pouyet et Moissette, 64, pl. 9, fig. 8.

Tortonien: Rio Vinalopo. Tortonien supérieur: El Plomo, Fortuna. Pliocène: Cuatro Calas, Santa Pola, Los Terreros.

Famille Microporellidae Hincks, 1880

Genre Microporella Hincks, 1877

Microporella ciliata (Pallas, 1766)

Fig. $2 \mathrm{f}$

1766 Eschara ciliata Pallas, 38.

1988 Microporella ciliata (Pal.); Zabala et Maluquer, 141, fig. 337.

1992 Microporella ciliata (Pal.); Pouyet et Moissette, 64, pl. 9, fig. 11.
1996 Microporella ciliata (Pal.); Haddadi-Hamdane, 91, pl. 7, fig. 3 .

Tortonien supérieur: El Plomo, Fortuna, Sierra Gorda. Pliocène: Los Terreros, Santa Pola.

Microporella coronata (Savigny-Audouin, 1826)

Fig. $2 \mathrm{a}-\mathrm{b}$

1826 Flustra coronata Savigny Audouin, 329, pl. 9, fig. 6.

1988 Microporella coronata (S.-A.); Moissette, 151, pl. 24, fig. 2-3.

1992 Microporella coronata (S.-A.); Pouyet et Moissette, 65 , pl. 9 , fig. 12.

Tortonien supérieur: Fortuna. Messinien: Carboneras. Pliocène: Santa Pola.

Genre Calloporina Neviani, 1895

Calloporina decorata (Reuss, 1848)

1848 Cellepora decorata Reuss, 89, pl. 10, fig. 25.

1996 Calloporina decorata (R.); Haddadi-Hamdane, 94, pl. 7, fig. 4.

1997b Calloporina decorata (R.); Pouyet, 68, pl. 7, fig. 10.

Tortonien: Rio Vinalopo. Tortonien supérieur: El Plomo, Fortuna, Sierra Gorda. Messinien: Sierra Alhamilla. Pliocène: Cuatro Calas, Los Terreros.

Genre Diporula Hincks, 1879

Diporula verrucosa (Peach, 1868)

1868 Eschara verrucosa Peach, 116.

1988 Diporula verrucosa (Peach); Zabala et Maluquer, 137, text-fig. 323, pl. 19, fig. B..

1992 Diporula verrucosa (Peach); Pouyet et Moissette, 66, pl. 10, fig. 1-2.

1992 Diporula verrucosa (Peach); Reguant et Maluquer, 150 , pl. 2, fig. 3 .

1996 Diporula verrucosa (Peach); Haddadi-Hamdane, 93, pl. 8 , fig. 2,5 ; pl. 9, fig. 7.

Tortonien supérieur: El Plomo. Pliocène: Cuatro Calas.

Genre Fenestrulina Jullien, 1880

Fenestrulina harmelini David, Mongereau et Pouyet, 1972

1972 Fenestrulina harmelini David, Mongereau et Pouyet, 67, pl. 2, fig. 5-7.

1988 Fenestrulina harmelini D. et P.; Moissette, 153, pl. 24, fig. 6.

1992 Fenestrulina harmelini D. et P.; El Hajjaji, 223, pl. 11, fig. 4.

Pliocène: Santa Pola.

Famille Escharellidae Levinsen, 1909

Genre Escharella Gray, 1848 


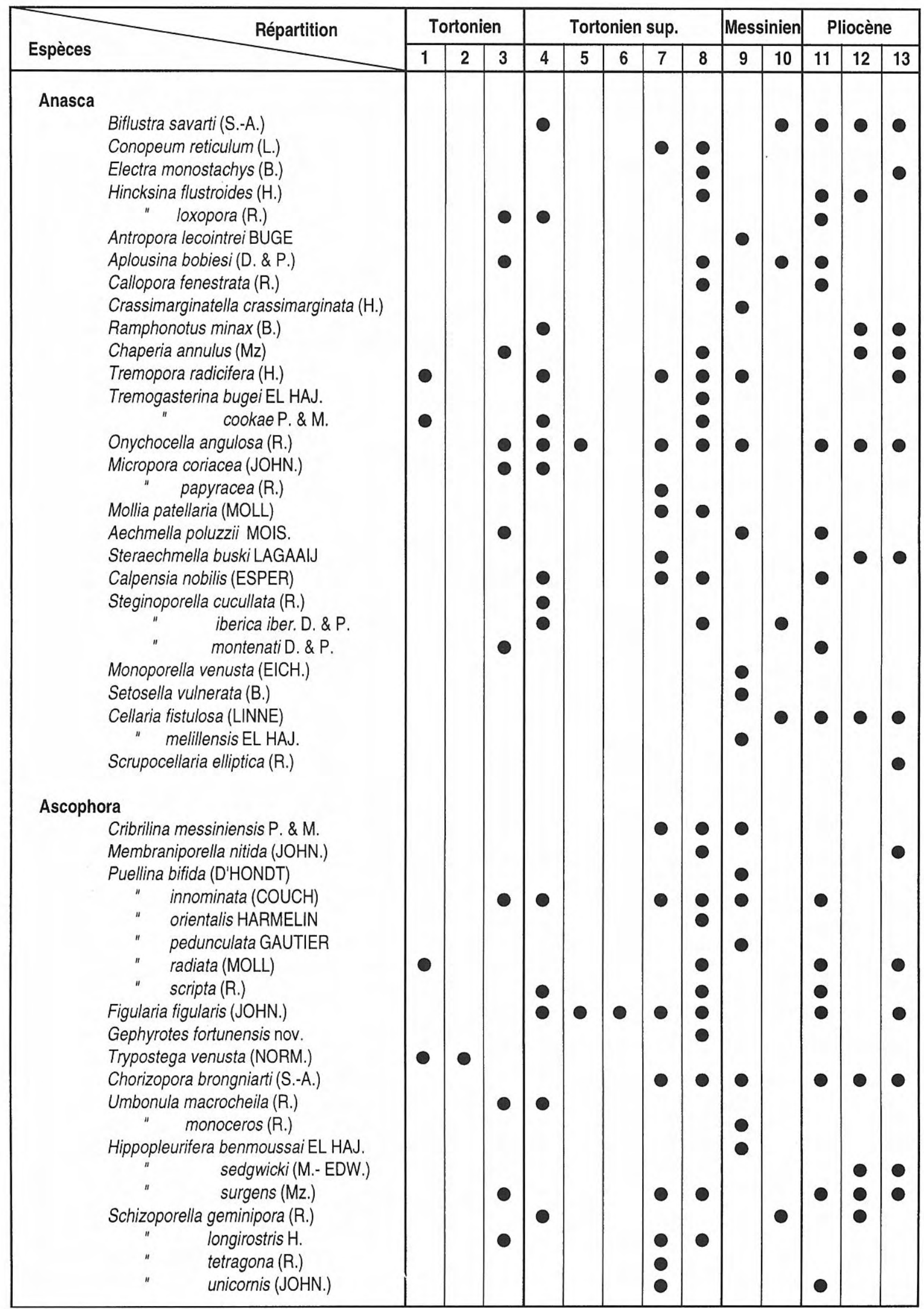

Figure 4. Répartition des espèces dans les 13 gisements étudiés. 1 Abanilla, 2 Campules, 3 Rio Vinalopo, 4 El Plomo, 5 Cabo Gata, 6 Crevillente, 7 Sierra Gorda, 8 Fortuna, 9 Carboneras, 10 Sierra Alhamilla, 11 Santa Pola, 12 Los Terreros, 13 Cuatro Calas. 


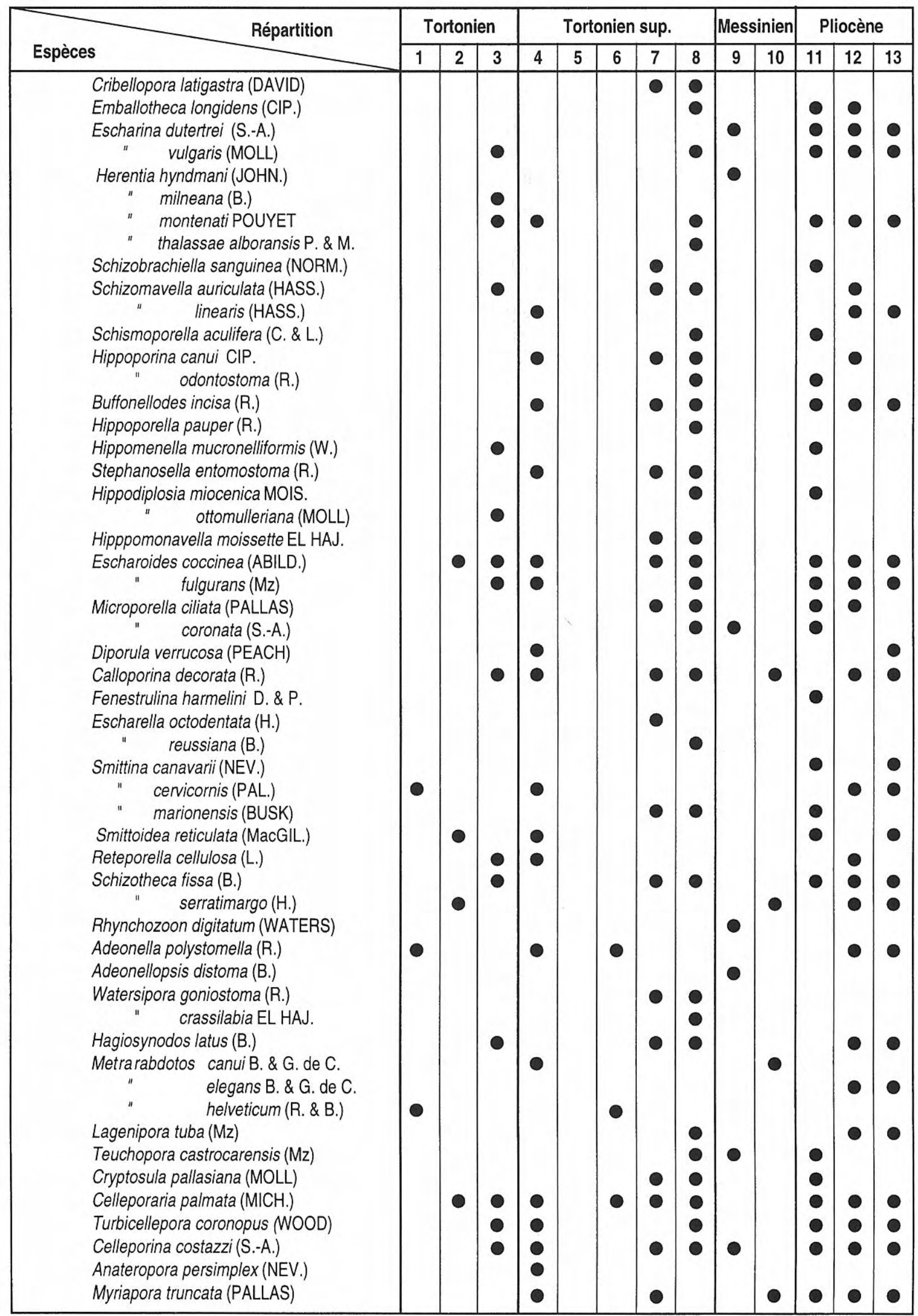

(continuation) 


\section{Escharella octodentata (Hincks, 1880)}

1880 Mucronella peachii var. ß (octodentata) Hincks, 361, pl. 51, fig. 2 .

1992 Escharella octodentata (Hincks); El Hajjaji, 184, pl. 13 , fig. 5 .

1997a Escharella octodentata (Hincks); Pouyet, 31, fig. 22.

Tortonien supérieur: Sierra Gorda.

\section{Escharella reussiana (Busk, 1859)}

1859 Lepralia reussiana Busk, 53, pl. 8, fig. 2.

1989 Escharella reussiana (Busk); Bishop et Hayward, 32, figs. 132-133.

1992 Escharella reussiana (Busk); El Hajjaji, 185, pl. 13, fig. 8 .

Tortonien supérieur: Fortuna.

Genre Smittina Norman, 1903

Smittina canavarii (Neviani, 1900)

1900 Smittia Canavarii Neviani, 368, fig. 5.

1975 Smittina canavarii (Nev.); Poluzzi, 62, text-fig. 4; pl. 19, fig. 5-6; pl. 21, fig. 10-11.

1976 Smittina canavarii (Nev.); Pouyet, 71, pl. 11, fig. 6.

1992 Smittina canavarii (Nev.); El Hajjaji, 173, pl. 10, fig. 4-5.

1992 Smittina canavarii (Nev.); Pouyet et Moissette, 69, pl. 10 , fig. 8 .

Pliocène: Cuatro Calas, Santa Pola.

Smittina cervicornis (Pallas, 1766)

1766 Millepora cervicornis Pallas, 252.

1988 Porella cervicornis (Pal.); Zabala et Maluquer, 121, fig. 266; pl. 12, fig. C.

1989 Smittina cervicornis (Pal.); Bishop et Hayward, 28, figs. 114-116; 30, figs. 117-118.

1992 Smittina cervicornis (Pal.); El Hajjaji, 174, pl. 10, fig. 8.

1997b Smittina cervicornis (Pal.); Pouyet, 70, pl. 7, fig. 9.

Tortonien: Abanilla. Tortonien supérieur: El Plomo. Pliocène: Cuatro Calas, Los Terreros.

\section{Smittina marionensis (Busk, 1854)}

1854 Lepralia marionensis Busk, 67, pl. 96, fig. 1-2.

1988 Smittina aff. marionensis (Busk); Moissette, 162, pl. 26, fig. 8 .

La frontale percée de trémopores, le péristome net, le petit aviculaire labial, l'ovicelle à frontale perforée et les dimensions nous font attribuer nos spécimens à l'espèce de Busk.

Tortonien supérieur: Fortuna, Sierra Gorda. Pliocène: Santa Pola.
Genre Smittoidea Osburn, 1952

Smittoidea reticulata (MacGillivray, 1842)

1842 Lepralia reticulata MacGillivray, 467.

1988 Smittoidea reticulata (MacG.); Zabala et Maluquer, 123, fig. 272; pl. 12, fig. D.

Tortonien: Campules. Tortonien supérieur: El Plomo. Pliocène: Cuatro Calas, Santa Pola.

Famille Reteporidae Smitt, 1867

Genre Reteporella Busk, 1884

( = Sertella Jullien, 1903)

Reteporella cellulosa (Linné, 1767)

1767 Millepora cellulosa Linné, 1284.

1992 Sertella cellulosa (L.); El Hajjaji, 235, pl. 14, fig. 12.

1992 Sertella cellulosa (L.); Pouyet et Moissette, 72, pl. 11, fig. 8.

Nous renvoyons au travail de Gordon (1984) pour la discussion concernant la synonymie des genres Reteporella et Sertella.

Tortonien: Rio Vinalopo. Tortonien supérieur: El Plomo. Pliocène: Los Terreros.

Genre Rhynchozoon Hincks, 1895

Rhynchozoon digitatum (Waters, 1879)

Fig. 3 b

1879 Cellepora digitata Waters, 197, pl. 14, fig. 13.

1962 Rhynchozoon digitatum (Waters); Gautier, 241.

1988 Rhynchozoon pseudodigitatum nov. sp., Zabala et Maluquer, 149, figs 384-387; pl. 20, figs E-F; pl. 21, fig. A.

Zoarium constitué de lamelles unilamellaires contournées donnant un aspect "massif" au zoarium. Une rangée de petits pores arrondis bordent chaque zoécie. Aperture semicirculaire. Dans les parties protégées de la colonie, on a pu observer devant l'aperture un péristome dressé, divisé en lobes digités ( 2 à 4 ) et portant sur sa face interne un aviculaire triangulaire labial. En plus, présence constante sur chaque zoécie d'un petit aviculaire frontal arrondi ou allongé, certaines cellules en portent deux. Ovicelle immergée, à frontale plane, inclinée au-dessus de l'aperture.

Remarque: En 1988, Zabala et Maluquer proposent un nouveau nom pour l'espèce décrite par Gautier qui pourtant avait contrôlé sa détermination. Il n'y a pas de désignation claire d'un holotype. Comme cela a été suggéré par Hayward (1974) une révision et une redéfinition des espèces méditerranéennes est nécessaire. Nous maintenons l'espèce digitatum en attendant cette révision.

Messinien: Carboneras.

Genre Schizotheca Hincks, 1877 


\section{Schizotheca fissa (Busk, 1856)}

1988 Schizotheca fissa (Busk); Zabala et Maluquer, 150, fig. 392, pl.23 A.

1992 Schizotheca fissa (Busk); Pouyet et Moissette, 71, pl. 11, fig. 1, 5 .

1996 Schizotheca fissa (Busk); Haddadi-Hamdane, 103, pl. 9 , fig. 5 .

Tortonien: Rio Vinalopo. Tortonien supérieur: Fortuna, Sierra Gorda. Pliocène: Cuatro Calas, Los Terreros, Santa Pola.

\section{Schizotheca serratimargo (Hincks, 1886)}

1886 Schizoporella serratimargo Hincks, 268, pl. 10, fig. 6. 1988 Schizotheca serratimargo (H.); Zabala et Maluquer, 150, fig. 393-394; pl.22 E-F.

1992 Schizotheca serratimargo (H.); El Hajjaji, 239, pl. 15, fig. 13.

1992 Schizotheca serratimargo (H.); Pouyet et Moissette, 72, pl. 11, fig. $2,3$.

1993 Schizotheca serratimargo (H.); Moissette et al., 109, fig. 7 d-e.

1996 Schizotheca serratimargo (H.); Haddadi-Hamdane, 104, pl. 9, fig. 4, 6 .

Tortonien: Campules. Messinien: Sierra Alhamilla. Pliocène: Cuatro Calas, Los Terreros.

Famille Adeonidae Jullien, 1903

Genre Adeonella Busk, 1884

Adeonella polystomella (Reuss, 1848)

pars 1848 Eschara polystomella Reuss, 70, pl. 8, fig. 27 (? fig. 28).

1989 Adeonella polystomella (R.); Schmid, p. 34, pl. 9 , fig. 3, 4, 6 .

1992 Adeonella polystomella (R.); Pouyet et Moissette, 73, pl. 11, fig. 6 .

1992 Adeonella polystomella (R.); El Hajjaji, 226, pl. 13, fig. 13.

Le spécimen de la planche 8, fig 28 (Reuss, 1848) figuré par Reuss provient non du Miocène moyen du Bassin de Vienne, Autriche mais de l'Eocène supérieur du Val di Lonte, Italie. Schmid (1989) met en doute l'appartenance de ce spécimen à l'espèce $A$. polystomella.

Tortonien: Abanilla. Tortonien supérieur: El Plomo, Sierra de Crevillente. Pliocène: Cuatro Calas, Los Terreros.

\section{Genre Adeonellopsis MacGillivray, 1886} Adeonellopsis distoma (Busk, 1858)

Fig. 3 a

1858 Lepralia distoma Busk, 127, pl. 18, fig.1.

1962 Adeonellopsis distoma (Busk); Gautier, 221.

1970 Adeonellopsis distoma (Busk); d'Hondt, 241, fig. 7.

1985 Adeonellopsis distoma (Busk); Aristegui, 425, fig. 2.
1992 Adeonellopsis distoma (Busk); Reguant et Maluquer, 145 , pl. 1 , fig. 9 .

Quelques petits fragments remarquablement bien conservés. Zoarium bilamellaire, en frondes étroites. Zoécies alternes, elliptiques. Une rangée de petits pores aréolaires arrondis bordent chaque zoécie. Frontale avec fort bourrelet entourant une dépression frontale perforée: le nombre et la disposition des perforations sont variables d'une cellule à l'autre. Aperture semi-circulaire. Sur notre matériel, chaque zoécie porte un aviculaire frontal sous-oral, triangulaire, vertical ou lègèrement oblique. A la base de quelques zoécies, présence de petits aviculaires interzoéciaux également triangulaires et obliques.

Messinien: Carboneras.

Cette espèce est connue en Méditerranée (Sicile, Tunisie, golfe de Gênes, Hyères, Alboran) et en Atlantique de la Bretagne aux Açores, à des profondeurs de $100 \mathrm{~m}$ à $350 \mathrm{~m}$. Dans le domaine fossile, elle est citée dans le Pliocène du domaine nordique, le Plio-Pléistocène de Rhodes et l'Holocène du Sud de l'Espagne.

\section{Famille Cheiloporinidae Bassler, 1936 Genre Watersipora Neviani, 1895}

Le genre Watersipora est synonyme du genre Dakaria Jullien, 1903, il a donc priorité (Soule et al., 1995, p. 188).

Watersipora crassilabia (El Hajjaji, 1987)

1987 Dakaria crassilabia El Hajjaji, 691, pl. 1, fig. 5-6.

1992 Dakaria crassilabia El Hajj.; El Hajjaji, 198, pl. 12, fig. 7.

Tortonien supérieur: Fortuna.

Watersipora goniostoma (Reuss, 1848)

1848 Cellepora goniostoma Reuss, 87, pl. 10, fig. 18.

1992 Dakaria goniostoma (R.); Pouyet et Moissette, 55, pl. 7, fig. 12.

1997b Watersipora goniostoma (R.); Pouyet, 75, pl. 8, fig. 5.

Tortonien supérieur: Fortuna, Sierra Gorda.

Famille Cleidochasmatidae Cheetham et Sandberg, 1964 Genre Hagiosynodos Bishop et Hayward, 1989 Hagiosynodos latus (Busk, 1856)

1989 Hagiosynodos latus (Busk); Bishop et Hayward, 46, figs. 189-191.

1992 Hippopodinella lata (Busk); Pouyet et Moissette, 75, pl . 11, fig. 11.

1996 Hippopodinella lata (Busk); Haddadi-Hamdane, 110, pl. 10 , fig. 9 . 
1997b Hagiosynodos latus (Busk); Pouyet, 76, pl. 8, fig. 8.

Tortonien: Rio Vinalopo. Tortonien supérieur: Fortuna, Sierra Gorda Pliocène: Cuatro Calas, Los Terreros.

Famille Metrarabdotosidae Vigneaux, 1949 Genre Metrarabdotos Canu, 1914

Metrarabdotos canui Buge et Galopim de Carvalho, 1963 Fig. $3 \mathrm{~g}-\mathrm{h}$

1963 Metrarabdotos canui Buge et Galopim de Carvalho, 170, fig. 18-19; pl. 2, fig. 2-3.

1992 Metrarabdotos canui B. et G. C.; El Hajjaji, 229, pl. 14, fig. 5 .

1996 Metrarabdotos canui B. et G. C.; Haddadi-Hamdane, 106, pl. 10, fig. $1,2$.

Cette espèce se reconnaît par ses aviculaires oraux latéraux très petits et son ovicelle en forme de mitre à surface costulée.

Tortonien supérieur: El Plomo. Messinien: Sierra Alhamilla.

\section{Metrarabdotos elegans Buge et Galopim de Carvalho,} 1963

1963 Metrarabdotos elegans Buge et Galopim de Carvalho, 162, fig. 13-14 et pl. 1, fig. 3-4.

1976 Metrarabdotos elegans B. et G. C.; Pouyet, 74, pl. 12, fig. 1, 2, 7 .

1996 Metrarabdotos elegans B. et G. C.; Haddadi-Hamdane, 107 , pl. 10 , fig. 5 .

Pliocène: Cuatro Calas, Los Terreros.

\section{Metrarabdotos helveticum (Roger et Buge, 1947)}

1947 Trigonopora monilifera var. helvetica Roger et Buge, 228, fig. 4b.

1968 Metrarabdotos (Porometra) helveticum helveticum ( $\mathrm{R}$. et B.); Cheetham, 99, pl. 12, figs. 2-5, pl. 17, figs. 6-7.

Tortonien: Abanilla. Tortonien supérieur: Sierra de Crevillente

Famille Phylactellidae Canu et Bassler, 1917

Genre Lagenipora Hincks, 1877

Lagenipora tuba (Manzoni, 1875)

1875 Lepralia tuba Manzoni, 33, pl. 4, fig. 52-52a.

1976 Lagenipora tuba (Manz.); Pouyet, 75, pl. 12, fig. 4 ; pl. 13, fig. 4.

1988 Lagenipora tuba (Manz.); Moissette, 182, pl. 28, fig. $6,9$.

1992 Lagenipora tuba (Manz.); El Hajjaji, 244, pl. 15, fig. 5.

1992 Lagenipora tuba (Manz.); Pouyet et Moissette, 76, pl. 12 , fig. 6.

Tortonien supérieur : Fortuna. Pliocène: Cuatro Calas,
Los Terreros.

Famille Teuchoporidae Harmer, 1957

Genre Teuchopora Neviani, 1895

Teuchopora castrocarensis (Manzoni, 1875)

1875 Alecto castrocarensis Manzoni, 40, pl. 6, fig. 71.

1977 Teuchopora castrocarensis (Manz.); Poluzzi, 71, pl. 1, fig. 1-7.

1992 Teuchopora castrocarensis (Manz.); Pouyet et Moissette, 77, pl. 11, fig. 7.

1996 Teuchopora castrocarensis (Manz.); HaddadiHamdane, 113, pl. 10, fig. 8.

Tortonien supérieur: Fortuna.Messinien: Carboneras. Pliocène: Santa Pola.

Famille Cryptosulidae Vigneaux, 1949

Genre Cryptosula Canu et Bassler, 1925

Cryptosula pallasiana (Moll, 1803)

Fig. $2 \mathrm{c}$

1803 Eschara pallasiana Moll, 57, pl. 3, fig. 13 A-B.

1992 Cryptosula pallasiana (Moll); Gordon et Mawatari, 29, pl 1 A-C; pl. 3 C; pl. 8 C.

1996 Cryptosula pallasiana (Moll); Haddadi-Hamdane, 111, pl. 10 , fig. 3.

Tortonien supérieur: Fortuna, Sierra Gorda. Pliocène: Santa Pola.

Famille Celleporariidae Harmer, 1957

Genre Celleporaria Lamouroux, 1821

Celleporaria palmata (Michelin, 1847)

1847 Cellepora palmata Michelin, 325, pl. 78, fig. 1a, b.

1996 Celleporaria palmata (Mich.); Haddadi-Hamdane, 114 , pl. 11 , fig. $3,5,7$ et 9 .

Tortonien: Campules, Rio Vinalopo. Tortonien supérieur: El Plomo, Fortuna, Sierra de Crevillente, Sierra Gorda. Pliocène: Cuatro Calas, Los Terreros, Santa Pola.

Famille Celleporinidae Busk, 1852

Genre Turbicellepora Ryland, 1963

Turbicellepora coronopus (Wood, 1844)

1844 Cellepora coronopus Wood, 18.

1996 Turbicellepora coronopus (Wood); Haddadi-Hamdane, 117 , pl. 12, fig. 7,8 .

1997b Turbicellepora coronopus (Wood); Pouyet, 80, pl. 8, fig. 10-12.

Tortonien: Rio Vinalopo.Tortonien supérieur: Fortuna, El Plomo. Pliocène: Cuatro Calas, Los Terreros, Santa Pola.

Genre Celleporina Gray, 1848

Celleporina costazi (Savigny-Audouin, 1826) 
1996 Celleporina costazi (Sav.-Aud.); Haddadi-Hamdane, 115, pl. 12, fig. 5 .

1997b Celleporina costazi (Sav.-Aud.); Pouyet, 79, pl. 8, fig. 9.

Tortonien: Rio Vinalopo. Tortonien supérieur: El Plomo, Fortuna, Sierra Gorda. Messinien: Carboneras. Pliocène: Cuatro Calas, Los Terreros, Santa Pola.

Famille Mamilloporidae Canu et Bassler, 1927

Genre Anoteropora Canu et Bassler, 1927

Anoteropora persimplex (Neviani, 1895)

1895 Stichoporina persimplex Neviani, 249, fig. 5.

1992 Anoteropora persimplex (Nev.); El Hajjaji, 233, pl. 14, fig. 10-11.

Tortonien supérieur: El Plomo.

Famille Myriaporidae Gray, 1841

Genre Myriapora de Blainville, 1830

Myriapora truncata (Pallas, 1766)

1766 Millepora truncata Pallas, 249.

1992 Myriapora truncata (Pallas); El Hajjaji, 215, pl. 15, fig. 7.

Tortonien supérieur: El Plomo, Sierra Gorda. Messinien: Sierra Alhamilla. Pliocène: Cuatro Calas, Los Terreros, Santa Pola.

\section{CONCLUSION}

Cette étude n'est pas exhaustive car elle n'inclut pas les cyclostomes. Il s'agit d'un travail essentiellement systématique, première étape qui nous a semblé nécessaire devant la pauvreté des données sur les bryozoaires néogènes de ces bassins. La figure 4 donne la répartition des espèces dans les 13 gisements étudiés.

Le matériel d'El Plomo a été récolté sur la plage dans une calcarénite bioclastique et lithoclastique transgressant sur le substratum volcanique (De La Chapelle, 1988, p. 62). La macrofaune est relativement abondante et variée: bryozoaires, gorgonaires isididés, crinoïdes, polypiers ahermatypiques. La microfaune est représentée par des foraminifères benthiques et néritiques et quelques rares formes planctoniques. Ces organismes sont issus de bathymétries très différentes. Ainsi les crinoïdes étudiés par Roux et Montenat (1977) donnent, par comparaison avec les formes actuelles proches, une bathymétrie allant de - 100 à - $700 \mathrm{~m}$ avec un pic vers - $500-600 \mathrm{~m}$. Les bryozoaires sont des formes de plate-forme, le gisement d'El Plomo étant situé à proximité d'une ligne de dislocation importante, l'accumulation des matériaux volcaniques a provoqué l'édification d'un relief sousmarin à forte pente; il est donc probable que les organismes benthiques ont glissé sur cette pente.

La faune de Carboneras est une thanatocénose très diversifiée: coraux au sens large, spongiaires, brachiopodes, bryozoaires, cirripèdes, mollusques, échinodermes, serpules et poissons. Elle est considérée comme appartenant au milieu bathyal supérieur. Rosso avait établi une liste préliminaire des bryozoaires (Rosso in Barrier et al., 1992) généralement limitée au niveau générique. Nous décrivons ici 22 espèces de cheilostomes dont 12 trouvées uniquement dans ce gisement; la plupart des spécimens encroûtent des hydrozoaires, des sclératiniaires ou des internodes d'Isididae (gorgonaires). L'état de conservation est excellent mais le matériel est très fragile.

Le gisement de Fortuna est le plus riche avec 55 cheilostomes. Il s'agit essentiellement de formes encroûtantes sur des huîtres ou des Porites. C'est un faciès périrécifal.

\section{LISTE BIBLIOGRAPHIQUE}

Abildgaard, P.D. 1806. In: Zoologica Danica seu animalium daniae et norvegiae. Müller ed., 1-46.

Aristegui, J. 1985. The genus Adeonellopsis MacGillivray (Bryozoa: Cheilostomata) in the Canary Islands: $A$. distoma (Busk) and A. multiporosa sp. nov. Journal of Natural History, London, 19, 425-430.

Audouin, V. 1828. Explication sommaire des planches de polypes de l'Egypte et de la Syrie publiées par JulesCésar Savigny, 225-244. In Description de l'Egypte, $2^{\circ}$ éd.,volume 23. Pancoucke édit., Paris.

Barrier, P., Zibrowius, H., Lozouet, P., Montenat, C., Ott D'Estevou, P., Serrano, F. et Soudet, H.-J. 1992. Une faune de fond dur du bathyal supérieur dans le Miocène terminal des Cordillères bétiques (Carboneras, SE Espagne). Mésogée, Marseille, 51, 3-13.

Bishop, J.D.D. 1986. The identity of Cribrilaria innominata (Couch, 1844) (Bryozoa, Cheilostomata). Bulletin of the British Museum (Natural History), Zoology ser., 50 (2), 93-102.

Bishop, J.D.D. and Hayward, P.J. 1989. SEM atlas of type and figured material from Robert Lagaaij's 'The Pliocene Bryozoa of the Low Countries' (1952). Mededelingen Rijks Geologische Dienst, 43 (2), 1-64.

Bishop, J.D.D. and Househam, B.C. 1987. Puellina (Bryozoa; Cheilostomata; Cribrilinidae) from British and adjacent waters. Bulletin of the British Museum (Natural History), Zoology ser., 53 (1), 1-63.

Brachert, T.C., Betzler, C., Braga, J.C. and Martin, J.M. 1996. Record of climatic change in neritic carbonates: turnover in biogenic associations and depositional modes (Late Miocene, southern Spain). Geologische Rundschau, 85, 327-337.

Buge, E. 1966. Sur quelques bryozoaires du Pliocène du Maroc. Annales de Paléontologie, Invertébrés, Paris, 52, 19-48.

Buge, E. et Galopim de Carvalho, A.M. 1963. Révision du genre Metrarabdotos Canu, 1914 (Bryozoa, Cheilostomata). Revista da Faculdade de Ciências de Lisboa, 2, C11, 137-196.

Busk, G. 1854. Catalogue of marine Polyzoa in the collection of the British Museum. Part II. Cheilostomata 
(part.). Catalogue of the British Museum (Natural History), 55-120.

Busk, G. 1858. On some Madeira Polyzoa. Quaterly Journal of Microscopical Science, London, 7, 65-67.

Busk, G. 1859. A monograph of the fossil Polyzoa of the Crag. Monograph of the Palaeontographical Society, London, 14, 1-136.

Busk, G. 1860. Descriptions of new species of polyzoa collected by Georges Barleem esq., in Shetland. Quaterly Journal of Microscopical Science, London, 8, 123-125.

Canu, F. et Lecointre, G. 1925. Bryozoaires Cheilostomes des faluns de Touraine et d'Anjou. Mémoires de la Société Géologique de France, N. S. 3, 2, 1-18.

Cheetham, A. H. 1968. Morphology and systematics of the Bryozoan genus Metrarabdotos. Smithsonian Miscellaneous Collections, Washington, 153 (1), 1-121.

Cipolla, F. 1921. I briozoi pliocenici di Altavilla presso Palermo. Giornale della Societa di Scienze Naturali ed Economoche di Palermo, 32, 163-330.

Couch, R.Q. 1844. A cornish fauna. 3. The Zoophytes and calcareous corallines. The Royal Institution of Cornwall, Truro, 1-164.

David, L. 1949. Quelques bryozoaires nouveaux du Miocène du Gard et de l'Hérault. Bulletin de la Société Géologique de France, sér. 5, 19, 539-544.

David, L., et Pouyet, S. 1972. Deux espèces de Steginoporella (Bryozoa, Cheilostomata) du Néogène d'Espagne. Geobios, 5, 237-245.

David, L. et Pouyet, S. 1974. Révision des bryozoaires cheilostomes miocènes du Bassin de Vienne, Autriche. Documents des Laboratoires de Géologie de la Faculté des Sciences de Lyon, 60, 83- 257.

David, L., Mongereau N. et Pouyet, S. 1972. Bryozoaires du Néogène du bassin du Rhône. Gisements burdigaliens de Mus (Gard). Documents des Laboratoires de Géologie de la Faculté des Sciences de Lyon, 52, 1-118.

De La Chapelle, G. 1988. Le bassin néogène de NijarCarboneras (Sud-Est de l'Espagne): les relations entre la sédimentologie et les étapes de la structuration. Thèse $\mathrm{n}^{\circ}$ 5088, Lyon,1-251 (inédit).

Eichwald, E.D. 1853. Lethaea rossica ou Paléontologie de la Russie. Stuttgart, 1-533.

El Hajjaji, K. 1987. Bryozoaires nouveaux du Messinien de Melilla. Geobios, 20, 687-694.

El Hajjaji, K. 1992. Les bryozoaires du Miocène supérieur du Maroc nord-oriental. Documents des Laboratoires de Géologie de Lyon, 123, 1-355.

Esper, E.J.C. 1796. Fortsetzungen der Pflanzenthiere in Abbildungen nach der Natur mit Farben erleuchtet nebst Beschreibung. Nüremberg, 1-230.

Gautier, Y.V. 1956. Résultats scientifiques des campagnes de la "Calypso" (fasc. II). V. Bryozoaires in "Etudes sur l'îlot du Grand Congloué". Annales de l'Institut océanographique, 32, 189-225.

Gautier, Y.V. 1962. Recherches écologiques sur les bryozoaires cheilostomes en Méditerranée occidentale. Recueil des Travaux de la station marine d'Endoume, Marseille, 24, 38, 1-434.

Gordon, D. P. 1984. The marine fauna of New Zealand: Bryozoa: Gymnolaemata from the Kermadec Ridge. New
Zealand Oceanographic Institute Memoir 91, 1-198.

Gordon, D. P. and Mawatari, S.F. 1992. Atlas of marine fouling Bryozoa of New Zealand ports and harbours. Miscellaneous Publications New Zealand Oceanographic Institute, 107, 1-52.

Haddadi-Hamdane, A. 1996. Bryozoaires du Pliocène du Sahel d'Alger. Documents des Laboratoires de Géologie de Lyon, 140, 1-189.

Harmelin, J.-G. 1970. Les Cribrilaria (Bryozoaires Chilostomes) de Méditerranée: systématique et écologie. Cahiers de Biologie marine, 11, 77-98.

Harmelin, J.-G. 1973. Les Bryozoaires des peuplements sciaphiles de Méditerranée: le genre Crassimarginatella Canu (Chilostomes Anasca). Cahiers de Biologie marine, Roscoff, 14, 471- 492.

Harmelin, J.-G. 1988. Espèces affines microsympatriques chez Puellina (Bryozoa, Cheilostomata) et description d'espèces nouvelles. Zoologica Scripta, 17, 25-38.

Harmelin, J.-G. and Aristegui, J. 1988. New Cribrilinidae (Bryozoa, Cheilostomata) from the upper bathyal of the Atlanto-Mediterranean region. Journal of Natural History, London, 22, 507-535.

Harmelin, J.-G. and Hondt, J.-L. d' 1993. Transfers of Bryozoan species between the Atlantic Ocean and the Mediterranean Sea via the Strait of Gibraltar. Oceanologica Acta, 16 (1), 63-72.

Hassall, A.H. 1842. Catalogue of Irish Zoophytes. The Annals and Magazine of Natural History, London, 6 , 166-175.

Hayward, P.J. 1974. Studies on the cheilostome bryozoan fauna of the Aegean island of Chios. Journal of Natural History, London, 8, 369-402.

Hayward, P.J. and Ryland, J.D.D. 1995. The British species of Schizoporella (Bryozoa: Cheilostomatida). Journal of Zoology, London, 237, 37-47.

Hincks, T. 1877. On British Polyzoa. The Annals and Magazine of Natural History, London, ser. 4, 20, 520532.

Hincks, T. 1880. A history of the British marine Polyzoa. Volume 1, $141+601$ p. Volume 2, atlas, 83 pl. Van Voorst, London.

Hincks, T. 1881. Contributions towards a general history of the marine Polyzoa. The Annals and Magazine of Natural History, London, ser. 5, 8, 1-14.

Hincks, T. 1886. The Polyzoa of the Adriatic: a supplement to Professor Heller's "Die Bryozoen des adriatischen meeres". The Annals and Magazine of Natural History, London, ser. 5, 17, 254-271.

Hondt, J.-L. d' 1970. Campagne d'essais du "Jean Charcot" (3-8 décembre 1968). 5. Bryozoaires. Bulletin du Muséum national d'Histoire naturelle, Paris, 42 (1), 232256.

Johnston, G. 1838. A history of the British Zoophytes. 1st edition. J. Strak, Edinburgh, 1-341.

Johnston, G. 1847. A history of the British Zoophytes. 2nd edition. Van Voorst, London, 1-488.

Lagaaij, R. 1952. The Pliocene Bryozoa of the Low Countries and their bearing on the marine stratigraphy of the North Sea region. Mededelingen van de Geologische Stichting, Maastricht, C5, 5, 1-233. 
Linné, C. 1758. Systemà Naturae. $10^{\circ}$ édition, Holmiae édt., Stockholm, 1, 1-824.

Linné, C. 1767. Systema Naturae. $12^{\circ}$ édition, Holmiae édt., Stockholm, 1, 533-1327.

MacGillivray, J. 1842. Catalogue of the marine zoophytes of the neighbourhood of Aberdeen. The Annals and Magazine of Natural History, London, 60, 462-469.

Manzoni, A. 1869. Briozoi fossili Italiani. Seconda contribuzione. Sitzungsberichten der Akademie des Wissenschaften Mathematische-Naturwissenschafte Klasse, Wien, 59, 512-523.

Manzoni, A. 1870. Briozoi fossili Italiani. Quarta contribuzione. Sitzungsberichten der Akademie des Wissenschaften Mathematische-Naturwissenschafte Klasse, Wien, 61, 323-349.

Manzoni, A. 1875. I briozoi del Pliocene antico di Castrocaro. Bologna, 1-64.

Michalzik, D. 1997. Sedimentationszyklen im Messinium (Tertiär, Ober-Miozän) SE-Spaniens. Neues Jahrbuch für Geologie und Paläontologie, Abhandlungen, 203 (1), 89143.

Michelin, H. 1841-1848. Iconographie zoophytologique. Bertrand édit, Paris, 1-348.

Milne Edwards, H. 1836. Observations sur les polypiers fossiles du genre Eschare. Annales des Sciences naturelles, Zoologie, (2), 6, 321-345.

Moissette, P. 1988. Faunes de bryozoaires du Messinien d'Algérie occidentale. Documents des Laboratoires de Géologie de Lyon, 102, 1-351.

Moissette, P. and Spjeldnaes, N. 1995. Plio-Pleistocene deep-water bryozoans from Rhodes, Grece. Palaeontology, 38, 771-799.

Moissette, P., Delrieu, B. et Tsagaris, S. 1993. Bryozoaires du bassin néogène d'Héraklion (Crète centrale, Grèce). Neues Jahrbuch für Geologie und Paläontologie, Abhandlungen, 190 (1), 75-123.

Moll, J.P.C. 1803. Eschara ex Zoophytorum seu Phytozoorum ordine pulcherimmum ac notatu dignissimum Genus novis speciebus auctum. Camesiniana ed., Wien, 1-70.

Montenat, C. 1977. Les bassins néogènes du Levant d'Alicante et de Murcia (Cordillères bétiques orientales, Espagne). Stratigraphie, Paléogéographie et Evolution dynamique. Documents des Laboratoires de Géologie de la Faculté des Sciences de Lyon, 69, 1-345.

Montenat, C. (Coord.) 1990. Les bassins néogènes du domaine bétique oriental (Espagne). Documents et Travaux de l'Institut géologique Albert-de-Lapparent, Paris, 12-13, 392 p., 3 cartes h.t. couleur.

Neviani, A. 1895. Briozoi fossili della Farnesina e Monte Mario presso Roma. Palaeontographica Italica, 1, 77140.

Neviani, A. 1900. Briozoi terziari e posterziari della Toscana. Bollettino della Società Geologica Italiana, 19 (2), 349-375

Norman, A. M. 1864. On undescribed british Hydrozoa, Actinozoa and Polyzoa. The Annals and Magazine of Natural History, London, ser. 3, 13, 82-90.

Norman, A.M. 1868. Notes on some rare british Polyzoa, with descriptions of new species. Quaterly Journal of
Microscopical Science, London, 8, 212-222.

Pallas, P.C. 1766. Elenchus Zoophytorum, sistens generum adumbrationes generaliores et specierum Cigniitarum succintas descriptiones, cum selectis auctorum synonymis. Van Cleef edit., La Haye, 1-451.

Peach, C.W. 1868. On a new british Eschara and the occurrence in Cornwall of Sphenotrochus wrightii. Journal of the royal Institution of Cornwall, 3, 116-117.

Poluzzi, A. 1975. I Briozoi cheilostomi del Pliocene della Val d'Arda (Piacenza, Italia). Memorie della Societa italiana di Scienze naturali e del Museo civico di Storia naturale di Milano, 21 (2), 37- 77.

Poluzzi, A. 1977. Contributi per una revisione del genere Teuchopora Neviani 1895 (bryozoa, Cheilostomata). Bollettino della Societa Paleontologica Italiana, 16, 6977.

Pouyet, S. 1976. Bryozoaires cheilostomes du Pliocène d'Aguilas (Espagne méridionale). Nouvelles Archives du Muséum d'Histoire naturelle de Lyon, 14, 53-82.

Pouyet, S. 1990. Les faunes de bryozoaires cheilostomes du Néogène bétique. In: Les bassins néogènes du domaine bétique oriental (Espagne) (Coord. C. Montenat). Documents et Travaux de l'Institut géologique Albert-deLapparent, Paris, 12-13, 49-51.

Pouyet, S. 1997a. Les bryozoaires du Pliocène de Normandie. Geodiversitas, Paris, 19 (1), 7-59.

Pouyet, S. 1997b. Les bryozoaires du Badénien (Miocène moyen) d'Olimpow (Pologne). Documents de Laboratoires de Géologie de Lyon, 145, 1-124.

Pouyet, S. et David, L. 1979. Révision sytématique du genre Steginoporella Smitt, 1873 (Bryozoa Cheilostomata). Geobios, 12, 763-817.

Pouyet, S. et Moissette, P. 1986. Bryozoaires cheilostomes nouveaux du Miocène supérieur du Bassin d'Alboran (Méditerranée occidentale). Geobios, 19, 385-391.

Pouyet, S. et Moissette, P. 1992. Bryozoaires du Pliocène d'Altavilla (Sicile-Italie): révision de la collection Cipolla, nouvelles données, paléoécologie. Palaeontographica, Stuttgart, A 223, 19-101.

Pouyet, S., El Hajjaji, K. and Mihraje, A. I. 1999. Lower Pliocene Bryozoa from Asilah (NW Morocco). Neues Jahrbuch für Geologie und Paläontologie, Abhandlungen, 213 (1), 119-144.

Reguant, S. 1961. Los briozoos del Neógeno español. Notas y Comunicaciones del Instituto Geológico y Minero de España, Madrid, 62, 215-244.

Reguant, S. 1993. The Cheilostoma Bryozoa from the Huelva Pliocene (SW Spain) in the Western Mediterranean context. Memorie di Scienze Geologiche, Padova, 45, 125-138.

Reguant, S. y Maluquer, P. 1992. Los Briozoos de los sedimentos superficiales holocenos de la plataforma continental del Cabo de Gata (Almería, España). Revista Española de Paleontología, 7, 141-153.

Reuss, A. 1848. Die fossilen Polyparien des Wiener Tertiärbeckens. Naturwissenschafte Abhandlungen von Haidinger, Wien, 2, 1-109.

Reuss, A. 1874. Die fossilen Bryozoen des österreichischungarischen Miocäns. Denks chriften der Kaiserlichen Akademie der Wissenschaften 
(Mathematisch-Naturwissenschafte Classe), Wien, 33 (1), 141-190.

Roger, J. et Buge, E. 1947. Les Bryozoaires du Redonien. Bulletin de la Société géologique de France, sér. 5, 16, 217-230.

Roux, M. et Montenat, C. 1977. Sites à Crinoüdes pédonculés et bathymétrie des bassins messiniens dans les cordillères bétiques orientales (Espagne méridionale). Bulletin de la Société géologique de France, sér. 7, 19 (2), 405-416.

Santisteban, C. and Taberner, C. 1983. Shallow marine and continental conglomerates derived from coral reef complexes after dessication of a deep marine basin: the Tortonian-Messinian deposits of the Fortuna basin, SE Spain. Journal of the Geological Society of London, 140, 401-411.

Sanz de Galdeano, C. 1990. Geologic evolution of the Betic Cordilleras in the Western Mediterranean, Miocene to present. Tectonophysics, 172, 107-119.

Savigny, J. 1826. Description de l'Egypte, Histoire naturelle. Planches, tome II. Panckoucke édit, Paris.

Schmid, B. 1989. Cheilostome Bryozoen aus dem Badenien (Miozän) von Nussdorf (Wien). Beiträge zur Paläontologie von Österreich, 15, 1-70.

Soule, D.F., Soule, J.D. and Chaney, H.W. 1995. The Bryozoa. In Taxonomic atlas of the benthic fauna of the Santa Maria Basin and Western Santa Barbara Channel. (Eds. Blacke, Chaney, Scott, Lissner) Santa Barbara Museum of Natural History, California, 13, 1-344.

Waters, A.W. 1879. On the Bryozoa (Polyzoa) of the Bay of Naples. The Annals and Magazine of Natural History, London, 5, 3, 192-202.

Waters, A.W. 1899. Bryozoa from Madeira. Journal of the royal microscopical Society of London, 3- 16.

Wood, S. V. 1844. Descriptive catalogue of the Zoophytes from the Crag. The Annals and Magazine of Natural History, London, ser. 1, 13, 10-21.

Zabala, M. and Maluquer, P. 1988. Illustrated keys for the classification of mediterranean Bryozoa. Treballs del Museu de Zoologia, Barcelona, 4, 1-294.

Manuscrito recibido: 28 de diciembre, 1999 Manuscrito aceptado: 25 de abril, 2000 